\title{
Observing Correlations Between Dark Matter Accretion and Galaxy Growth: II. Testing the Impact of Galaxy Mass, Star Formation Indicator, and Neighbour Colours
}

\author{
Christine O’Donnell ${ }^{1 \star}$, Peter Behroozi ${ }^{2}$, Surhud More ${ }^{3,4}$ \\ ${ }^{1}$ School of Earth \& Space Exploration, Arizona State University, Tempe, AZ 85281, USA \\ ${ }^{2}$ Department of Astronomy and Steward Observatory, University of Arizona, Tucson, AZ 85721, USA \\ ${ }^{3}$ Inter-University Centre for Astronomy and Astrophysics, Post bag 4, Ganeshkhind, Pune 411007, India \\ ${ }^{4}$ Kavli Institute for the Physics and Mathematics of the Universe, 5-1-5 Kashiwanoha, Kashiwa, 2778583, Japan
}

Accepted XXX. Received YYY; in original form ZZZ

\begin{abstract}
A crucial question in galaxy formation is what role new accretion has in star formation. Theoretical models have predicted a wide range of correlation strengths between halo accretion and galaxy star formation. Previously, we presented a technique to observationally constrain this correlation strength for isolated Milky Way-mass galaxies at $z \sim 0.12$, based on the correlation between halo accretion and the density profile of neighbouring galaxies. By applying this technique to both observational data from the Sloan Digital Sky Survey and simulation data from the UnIVERSEMACHINE, where we can test different correlation strengths, we ruled out positive correlations between dark matter accretion and recent star formation activity. In this work, we expand our analysis by (1) applying our technique separately to red and blue neighbouring galaxies, which trace different infall populations, (2) correlating dark matter accretion rates with $D_{n} 4000$ measurements as a longer-term quiescence indicator than instantaneous star-formation rates, and (3) analyzing higher-mass isolated central galaxies with $10^{11.0}<M_{*} / M_{\odot}<10^{11.5}$ out to $z \sim 0.18$. In all cases, our results are consistent with non-positive correlation strengths with $\gtrsim 85$ per cent confidence, which is most consistent with models where processes such as gas recycling dominate star formation in massive $z=0$ galaxies.
\end{abstract}

Key words: galaxies: formation - galaxies: haloes - galaxies:star-formation - dark matter

\section{INTRODUCTION}

According to the $\Lambda \mathrm{CDM}$ framework, galaxies form within dark matter haloes when gas gravitationally coalesces at halo centres (for reviews, see Somerville \& Davé 2015; Wechsler \& Tinker 2018). Thus, we expect that halo properties and galaxy properties will be strongly correlated, e.g., halo mass and stellar mass (Tinker et al. 2017b; Behroozi et al. 2019).

However, different models predict different correlation strengths between dark matter accretion and galaxy star formation. As material falls onto a halo from large distances, we expect the fraction of infalling gas versus infalling dark matter to match the cosmic baryon fraction. If this also holds true at smaller scales, then we would expect dark matter accretion and star formation to be correlated. For example, Wetzel \& Nagai (2015) found that dark matter accretes in a shell-like manner at $R_{200 \mathrm{~m}}$ around a halo. Gas, on the other hand, can radiatively cool, allowing it to decouple from

^ E-mail: Christine.ODonnell@asu.edu (CO) the dark matter and continue infalling onto the central galaxy. As a result, star formation rates track dark matter accretion rates (Wetzel \& Nagai 2015), and many theoretical models and simulations have found or assumed a perfect positive correlation strength between the two (e.g., Becker 2015; Rodríguez-Puebla et al. 2016a; Cohn 2017; Moster et al. 2018).

On the other hand, some models predict that feedback from winds, supernovae, AGN, and other processes will suppress new accretion onto central galaxies. Thus, most star formation is generated by recycled or re-accreted gas, and we would expect at most only a weak correlation with dark matter accretion (e.g., Kereš et al. 2005; Dekel \& Birnboim 2006; Nelson et al. 2013, 2015; Muratov et al. 2015; van de Voort 2017). Furthermore, Muratov et al. (2015) found that outflows from a galaxy (due to supernovae, AGN, etc.) are most significant at higher redshifts, creating an enriched gas reservoir that powers star formation at lower redshifts. These models are consistent with observational results that star formation rates do not correlate with major mergers (Behroozi et al. 2015), which have enhanced dark matter accretion rates. Further, Tinker et al. 
(2017a) studied SDSS galaxy groups and found that the fraction of quenched central galaxies with $M_{*} \gtrsim 10^{10.3} \mathrm{M}_{\odot}$ only slightly increases as the local environmental density increases. However, halo assembly rates are strongly correlated with local density (e.g., Lee et al. 2017), and so their results implied that halo growth and galaxy assembly are only weakly correlated.

In O'Donnell et al. (2021), we observationally constrained the correlation between dark matter accretion and recent star formation activity in Milky-Way mass galaxies $\left(10^{10.5}<M_{*} / \mathrm{M}_{\odot}<10^{11}\right)$. Our technique built on work to characterize the splashback radii of haloes, the radius at which newly accreted material reaches its first apocentre (e.g., Diemer \& Kravtsov 2014; More et al. 2015, 2016; Baxter et al. 2017). s a halo accretes more matter, its gravitational potential well deepens, which will tighten the orbits of satellite galaxies and thus steepen the halo's density profile. For more rapidly accreting haloes, their halo density profile steepens more strongly (Diemer \& Kravtsov 2014) and their splashback radii will decrease (More et al. 2015). Observational studies stacked the density profiles of nearby neighbours around thousands of clusters to look for the splashback feature by measuring excess galaxy counts around target clusters using background subtraction on photometric SDSS data (More et al. 2015, 2016; Baxter et al. 2017).

In our previous analysis, we also used neighbouring galaxies as probes of dark matter accretion. To measure correlations with galaxy star formation rates, we made two modifications to previous techniques. First, we selected Milky Way-mass galaxies, as star formation is still happening at these smaller mass scales (versus the centrals of galaxy clusters, which are often quenched). To reduce environmental contamination in neighbour density profiles, we specifically selected isolated Milky Way-mass galaxies. By selecting isolated galaxies that are the dominant source of gravity in their local environments, they will have stronger correlations between neighbouring galaxy orbits and dark matter accretion rates (see also Deason et al. 2020), which allows us to probe lower-mass halo scales than previous work. In addition, instead of identifying a single feature in the density profiles, we analyzed the shape of the entire neighbour density distribution to increase our signal-to-noise ratio. By comparing the measured shapes of the neighbour density distributions, our technique allows us to assess the dark matter accretion rates.

Our analysis compared observational SDSS DR16 data (Ahumada et al. 2019) to simulated UniverseMAchine data (Behroozi et al. 2019) to constrain the correlation strength. We separated starforming and quiescent isolated galaxies in the SDSS based on their specific star formation rates (SSFRs). Our results ruled out positive correlations between dark matter accretion rates and SSFRs with $\gtrsim 85$ per cent confidence.

This paper extends our previous work by ruling out several alternate interpretations of this finding. For example, we would expect weak correlations if the timescales probed by SSFRs are much shorter than the orbits of satellite galaxies $\left(\sim 2 t_{\text {dyn }} \sim 4\right.$ Gyr; see $\$ 5$ in O’Donnell et al. 2021). In this paper, we test two approaches that address this concern:

(i) Instead of only separating star-forming and quiescent galaxies based on their SSFRs, we also bin galaxies based on their $4000 \AA$ break $\left(D_{n} 4000\right.$; Balogh et al. 1999), which is a longer-term quiescence indicator.

(ii) We compare the density distributions of neighbouring galaxies based on the neighbours' colours. As a satellite galaxy falls into a host halo, gas is stripped from the satellite that would otherwise replenish star formation, leading to an increase in the fraction of red galaxies within host halo virial radii (e.g., Gunn \& Gott 1972; Moore et al. 1996; Dressler et al. 1997; Weinmann et al. 2006; Kawata \& Mulchaey 2008; Baxter et al. 2017). Wetzel et al. (2013) found that the typical timescale for this quenching is on the order of satellite orbital periods (2-4 Gyr). Because red satellites have been within their host haloes for a longer time, they may be more sensitive to changes in the gravitational potential well than blue satellites that have only recently fallen in. By analyzing the density distribution of red neighbours around isolated Milky Way-mass galaxies, we would have a more robust test of the correlation strength between dark matter accretion and star formation rates.

Furthermore, we expand our analysis to higher-mass isolated host galaxies. This test allows us to identify isolated central galaxies out to higher redshifts (up to $z<0.183$ versus $z<0.123$; see $\$ 2.1 .1$ for sample statistics), and it adds an additional check of our results by using an independent host population.

This paper is structured as follows: First, in $\S 2$, we summarise key details of our observational (\$2.1) and simulation data (\$2.2), including differences with the datasets used in O'Donnell et al. (2021). In §3, we describe the methodology used in our analysis. $\$ 4$ presents the results for separating star-forming and quiescent hosts based on SSFR versus $D_{n} 4000$ ( $\left.\$ 4.1\right)$, comparing the density distributions of red neighbours around isolated hosts to the distributions of blue neighbours ( $\$ 4.2$ ), and analysing higher-mass isolated hosts (§4.3). Finally, we conclude in $\$ 5$ and note directions for future analyses. We adopt a flat $\Lambda$ CDM cosmology with $\Omega_{M}=0.307$, $\Omega_{\Lambda}=0.693$, and $h=0.677$, consistent with Planck 2018 results (Planck Collaboration et al. 2018)

\section{OBSERVATIONS \& SIMULATIONS}

This paper uses similar techniques and datasets as in O'Donnell et al. (2021). Below, we repeat key details and note differences where appropriate.

\subsection{Observational Data}

We identify isolated galaxies, which we refer to as our isolated host sample (\$3), from the SDSS DR16 spectroscopic catalogs (Ahumada et al. 2019). We define isolated to mean that there is no larger galaxy within $2 \mathrm{Mpc}$ in projected (on-sky) physical distance or 1000 $\mathrm{km} / \mathrm{s}$ in velocity separation. We use median stellar masses, specific star formation rates, and $D_{n} 4000$ values from the MPA-JHU valueadded catalog (Kauffmann et al. 2003; Brinchmann et al. 2004). Stellar masses and star formation rates were converted to a Chabrier (2003) IMF by dividing each by a factor of 1.07 . To improve our isolated host selection, we supplemented these catalogs with data from the NYU Value-Added Galaxy Catalog (NYU-VAGC; Blanton et al. 2005) for galaxies with $M_{*}>10^{9.5} M_{\odot}$. The NYU-VAGC filled in information for galaxies affected by fibre collisions by assuming they have the same redshift as the nearest non-fibre-collided neighbour. Further, we excluded galaxies that are within $2 \mathrm{Mpc}$ of a survey boundary or region of significant incompleteness to ensure the robustness of our isolation criteria. To avoid Hubble flow corrections (e.g., Baldry et al. 2012), we exclude galaxies with $z<0.01$. Our resulting catalog has 547,271 galaxies over $6401.1 \mathrm{deg}^{2}$ of sky. Finally, we apply a stellar mass completeness cut to our spectroscopic catalog. Behroozi et al. (2015) found that in the SDSS, $>95$ per cent of galaxies have $r$-band apparent magnitudes $(r)$ brighter 
than the following limit:

$r<-0.25-1.9 \log _{10}\left(\frac{M_{*}}{M_{\odot}}\right)+5 \log _{10}\left(\frac{D_{L}(z)}{10 \mathrm{pc}}\right)$,

where $M_{*}$ is the stellar mass and $D_{L}$ is the luminosity distance given our cosmology. To be consistent with SDSS's spectroscopic survey limits, we exclude galaxies for which $r>17.77$ according to Eq. 1.

In this paper, we identified isolated host galaxies in two different mass bins: (1) galaxies with $10.5<\log _{10}\left(M_{*} / M_{\odot}\right)<11.0$ and (2) galaxies with $11.0<\log _{10}\left(M_{*} / M_{\odot}\right)<11.5$; the former is the same bin we used in O'Donnell et al. (2021).

To count neighbouring photometric galaxies around our isolated hosts, we use SDSS DR16 photometric catalogs (Ahumada et al. 2019). We use sources with a type field of 'GALAXY' to exclude likely stars, and we restrict our catalog to galaxies with $r<21.5$ to ensure reliability of $g-r$ colours. Following O'Donnell et al. (2021), we bin nearby neighbours by stellar mass to reliably compare the shape of density distributions around star-forming and quiescent hosts. We used the same fit between $g-r$ colours and $M_{*} / L_{r}$ ratios as found in O'Donnell et al. (2021):

$\log _{10}\left(M_{*} / L_{r}\right)=1.341(g-r)-0.639$.

In O'Donnell et al. (2021), we found that our results using this approach were consistent with those using luminosity binning (Appendix A1 of O'Donnell et al. 2021) and with using a fit between $g-r$ colours and mass from Bell et al. (2003) (Appendix A2 of O'Donnell et al. 2021).

To reduce noise when applying our fit, we cut our photometric catalog based on $g-r$ colours to exclude galaxies at higher redshifts. For isolated hosts with $10.5<\log _{10}\left(M_{*} / M_{\odot}\right)<11.0$, we restrict our analysis to galaxies with $0.0<g-r<1.0$, as redder galaxies are not present above background noise levels (Fig. 9 from O'Donnell et al. 2021) and tend to be at higher redshifts (e.g., Rykoff et al. 2014). These cuts result in a photometric catalog that includes 35,457,243 galaxies over an on-sky area of 18,509.0 deg ${ }^{2}$. We note that our results in O'Donnell et al. (2021) were not sensitive to the $g-r$ cutoff value; we obtained consistent results using a redder cutoff of $0.0<g-r<1.25$. The higher-mass isolated hosts $\left(11.0<\log _{10}\left(M_{*} / M_{\odot}\right)<11.5\right)$ can be detected at higher redshifts, so we use a limit of $g-r<1.25$ based on the colour distribution of nearby neighbours (Fig. 1), resulting in a photometric catalog that includes $47,713,412$ galaxies.

\subsubsection{Sample Statistics}

From O'Donnell et al. (2021), we identified 25,625 isolated galaxies from SDSS with stellar masses $10.5<\log _{10}\left(M_{*} / M_{\odot}\right)<11.0$ that correspond to a redshift range of $0.01<z<0.123$ (median $z=0.079$ ). In this paper, we also identify 25,432 isolated hosts with stellar masses $11.0<\log _{10}\left(M_{*} / M_{\odot}\right)<11.5$ (redshift range $0.01<z<0.183$, with median $z=0.116) .{ }^{1} \mathrm{We}$ also investigated using galaxies from a lower mass range $\left(10.0<\log _{10}\left(M_{*} / M_{\odot}\right)<\right.$ $10.5)$, but their neighbour density distributions were dominated by noise because there were too few isolated hosts even if we relaxed the isolation criteria (e.g., no larger galaxy within $1 \mathrm{Mpc}$ projected distance and $1000 \mathrm{~km} / \mathrm{s}$ velocity distance).

1 The isolated galaxy catalogs are available at https://github.com/ caodonnell/DM_accretion
To measure the uncertainties in neighbour density distributions, we used jackknife sampling. For each sample, a $\sim 10^{\circ} \times 10^{\circ}$ region was removed from the sky footprint $(\sim 37.5 \times 37.5 \mathrm{Mpc} / h$ at $z=0.079$ ), resulting in 112 samples with an average of $\sim 25,000$ isolated hosts in each mass bin per sample.

\subsubsection{Star Formation \& Quiescence Indicators}

In O'Donnell et al. (2021), we binned our isolated hosts into starforming and quiescent bins based on their specific star formation rates (SSFRs), which is an indicator of recent star formation activity. We separated the two SSFR bins at SSFR $=10^{-11} \mathrm{yr}^{-1}$ following Wetzel et al. (2012), and we keep the same definition here. As an additional test of our results, we attempted to separate the star-forming hosts into two bins since it is possible the correlation between star formation and dark matter accretion may differ for galaxies with stronger versus weaker star formation rates (Berti et al. 2021). However, even when separating the star-forming hosts into two bins by the median SSFR, we did not have sufficient signal-to-noise to identify differences in their neighbour density distributions.

As we noted in O'Donnell et al. (2021), the shape of the neighbour density distribution changes on timescales of satellite galaxy orbits $\sim 2 t_{\text {dyn }} \sim 4$ Gyr. If SSFRs change on shorter timescales than satellite galaxy orbits, then we would expect to see weaker correlations. To test this potential bias, we also split the isolated hosts into two bins based on their $4000 \AA$ break $\left(D_{n} 4000\right.$, Balogh et al. 1999 ), which is a longer-term indicator of quiescence. Kauffmann et al. (2003) found that SDSS spectroscopic data shows a bimodal distribution in $D_{n} 4000$. The first peak at $D_{n} 4000 \sim 1.3$ corresponds to galaxies with mean stellar ages $\sim 1-3 \mathrm{Gyr}$, and a second peak at $D_{n} 4000 \sim 1.85$ corresponds to galaxies with mean stellar ages $\sim 10$ Gyr. We see a similar distribution in our SDSS DR16 spectroscopic catalog (Fig. 2), and we split the star-forming and quiescent host galaxies at $D_{n} 4000=1.6$. This split is consistent with Kauffmann et al. (2003) and has been used in other analyses of SDSS galaxies (e.g., Blanton et al. 2011; Tinker et al. 2017a). We also investigated using a stellar mass-dependent cut between red and blue $D_{n} 4000$ galaxies following Geha et al. (2012), but it did not change our results. When binning isolated hosts by either SSFR or $D_{n} 4000$, we do not find any significant differences between the redshift distributions of the two bins.

As in O'Donnell et al. (2021), when using the star-forming fraction from SDSS to construct accretion rate correlation predictions in the simulation data from the UniverseMachine (\$3), we use the SDSS star-forming fraction among isolated hosts within 0.1 dex bins (e.g., from $\left.10.6<\log _{10}\left(M_{*} / M_{\odot}\right)<10.7\right)$. Fig. 3 plots the fraction of star-forming hosts for both star formation indicators across the isolated host mass ranges. The two indicators yield similar star-forming fractions across the isolated host mass range.

Finally, Fig. 4 compares the SSFR and $D_{n} 4000$ values for isolated hosts in both stellar mass bins. The two indicators track each other very well with $\lesssim 10$ per cent difference in isolated host classification. For isolated hosts with stellar masses $10.5<$ $\log _{10}\left(M_{*} / M_{\odot}\right)<11.0,7.3$ per cent of isolated hosts that are starforming based on their SSFR values are quiescent based on the $D_{n} 4000$ measurements, and 3.6 per cent of isolated hosts that are star-forming based on their $D_{n} 4000$ measurements are quiescent based on their SSFR values. Similarly, for isolated hosts with stellar masses $11.0<\log _{10}\left(M_{*} / M_{\odot}\right)<11.5$, 4.6 per cent of isolated hosts that are star-forming based on their SSFR values are quiescent based on the $D_{n} 4000$ measurements, and 2.0 per cent of isolated 

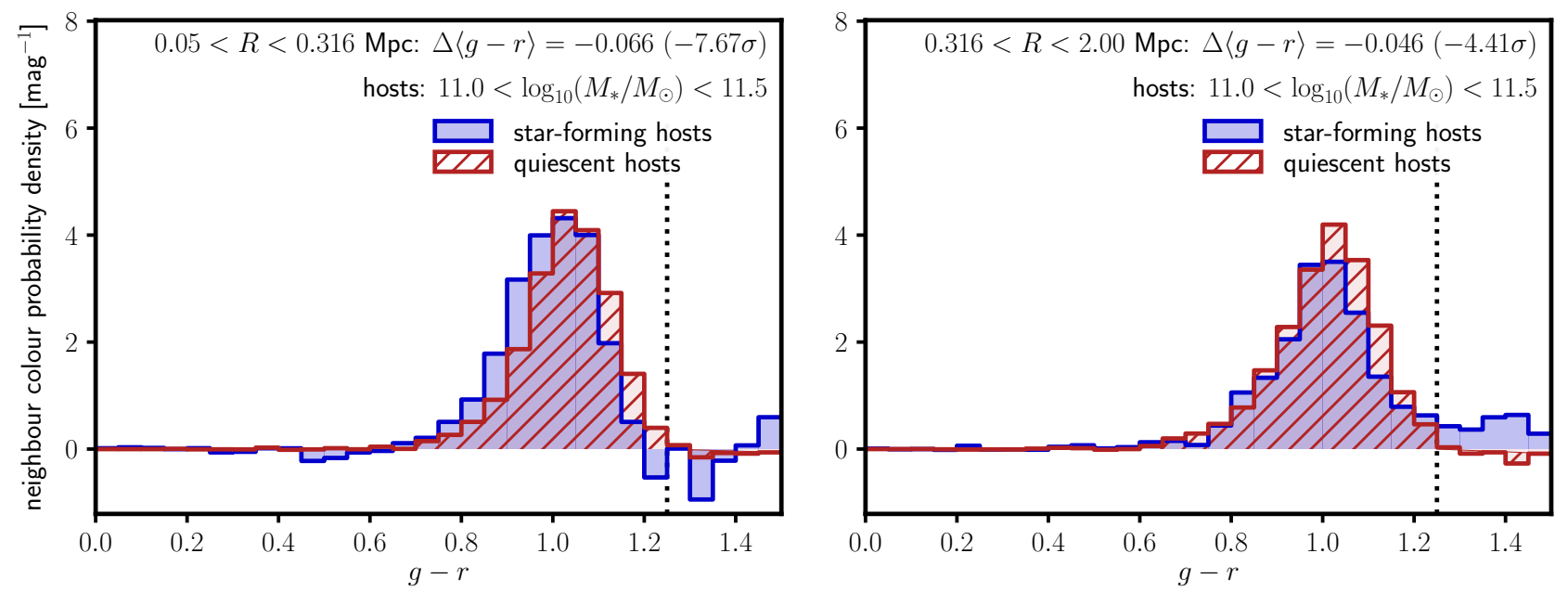

Figure 1. We exclude photometric galaxies with very red colours as they tend to be at higher redshifts (e.g., Rykoff et al. 2014); thus, applying a colour cut reduces noise in our neighbour density distributions. In O'Donnell et al. (2021), we excluded photometric galaxies with $g-r>1.0$ for isolated hosts with $10.5<\log _{10}\left(M_{*} / M_{\odot}\right)<11.0$ as these galaxies were not present above background noise counts. Here, we plot the background-subtracted weighted distribution of $g-r$ colours for our higher-mass isolated hosts $\left(11.0<\log _{10}\left(M_{*} / M_{\odot}\right)<11.5\right)$ and determine that the colour cut should be $g-r>1.25$ (indicated by the dotted vertical line). These plots include neighbours with $\log _{10}\left(M_{*} / M_{\odot}\right)>10.4$, which corresponds to the stellar mass limit at the maximum isolated host redshift $(z=0.183)$ given SDSS photometric limits. We note that lower-mass neighbours are expected to have bluer colours. The projected distance ranges of the two panels match the regions used in our analysis of the shapes of the neighbour density distributions (Eq. 5 in $\S 3$ ). Neighbours around star-forming hosts have bluer $g-r$ colours than neighbours around quiescent hosts, and the difference is more significant at closer distances from the hosts. We noted a similar difference in the neighbours around isolated hosts with $10.5<\log _{10}\left(M_{*} / M_{\odot}\right)<11.0$ (Fig. 9 in O'Donnell et al. 2021).

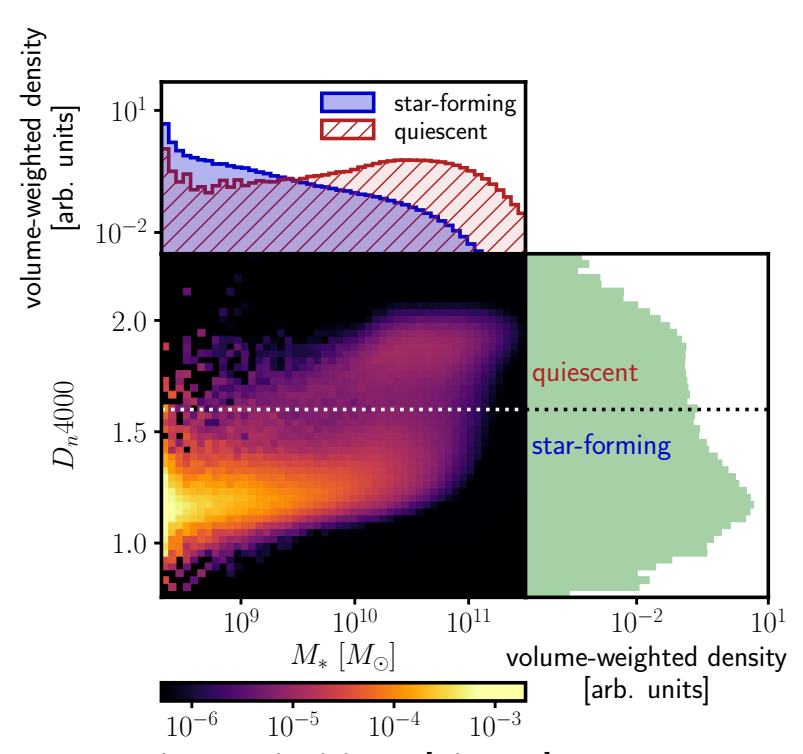

volume-weighted density [arb. units]

Figure 2. When using $D_{n} 4000$ as a star formation indicator, we bin our isolated hosts into star-forming and quiescent hosts split at $D_{n} 4000=1.6$. The central plot shows the volume-weighted density distribution of galaxies in the SDSS DR16 spectroscopic catalog. The top histogram shows the distribution of stellar masses of star-forming versus quiescent hosts based on their $D_{n} 4000$ values, and the right histogram shows the overall distribution of $D_{n} 4000$. Fig. 6 in O'Donnell et al. (2021) depicts analogous distributions when using specific star formation rates (SSFRs) as the star formation indicator.

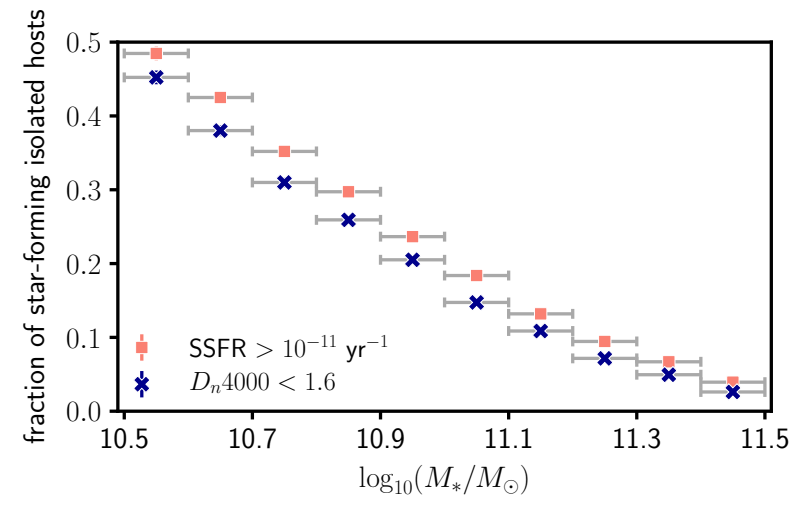

Figure 3. The fraction of star-forming isolated hosts in the SDSS is similar for both of the indicators used to bin star-forming versus quiescent hosts (specific star formation rates [SSFR] and $D_{n} 4000$ ) across the entire isolated host mass range. Each marker indicates the star-forming fraction for isolated hosts within a 0.1 dex bin (e.g., over $\left.10.7<\log _{10}\left(M_{*} / M_{\odot}\right)<10.8\right)$. The Poisson errors in the star-forming fractions are smaller than the sizes of the plot markers, and the grey horizontal bars indicate the width of the host stellar mass bins.

hosts that are star-forming based on their $D_{n} 4000$ measurements are quiescent based on their SSFR values.

\subsubsection{Red vs. Blue Neighbours}

As another validation of our approach, we bin neighbours by their $g-r$ colours and apply our analysis technique separately to each colour bin. As a satellite galaxy passes through the halo of its host galaxy, we expect that its star formation will quench. Galactic 

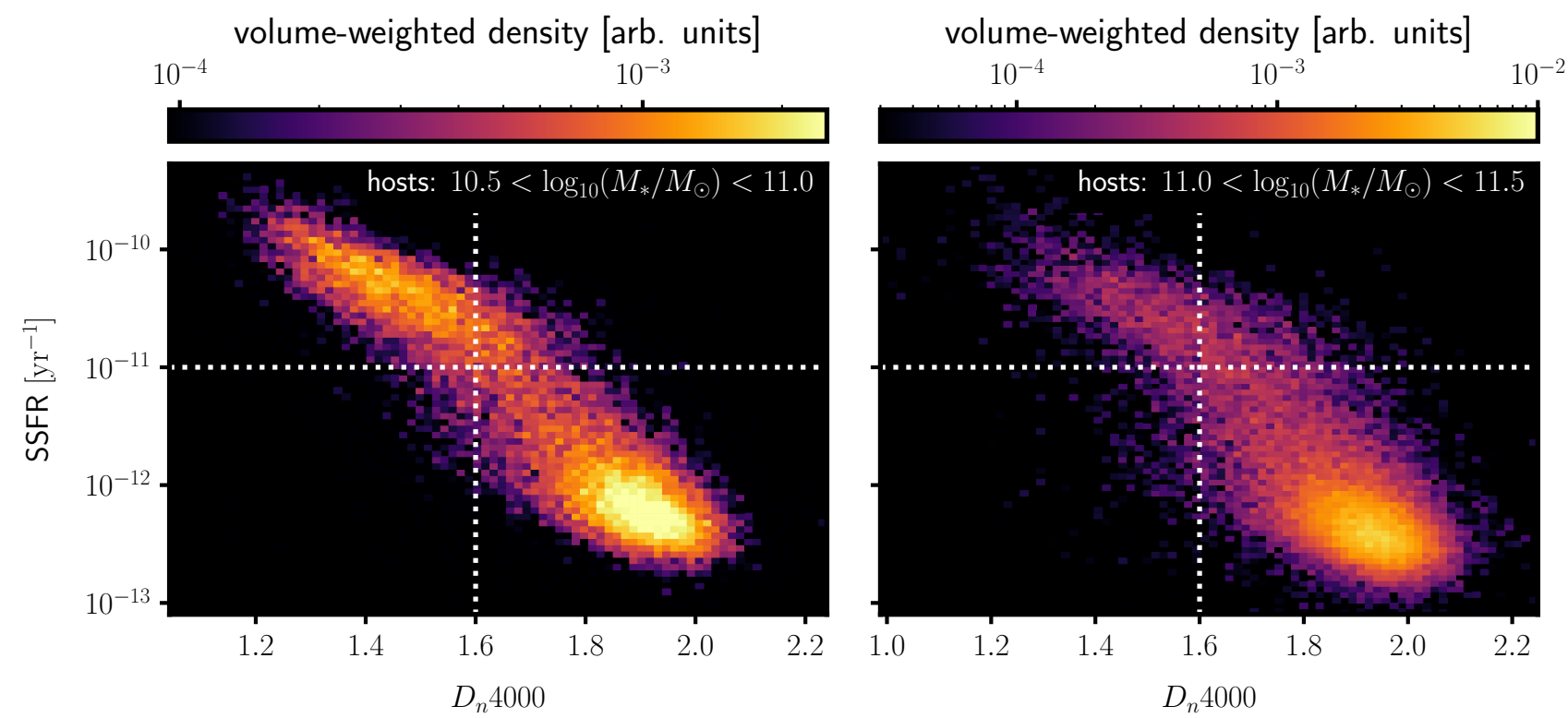

Figure 4. For isolated hosts in the SDSS, SSFR and $D_{n} 4000$ measurements yield consistent bins for star-forming versus quiescent hosts. The dotted vertical and horizontal lines indicate the values used to separate isolated host mass bins for each indicator (\$2.1.2). The left panel shows the distribution for isolated hosts with $10.5<\log _{10}\left(M_{*} / M_{\odot}\right)<11.0$, and only 10.9 per cent of hosts are classified differently between the two indicators (e.g., as star-forming by SSFR but quiescent by $\left.D_{n} 4000\right)$. The right panel shows the distribution for isolated hosts with $11.0<\log _{10}\left(M_{*} / M_{\odot}\right)<11.5$, and 6.6 per cent of hosts are classified differently between the two indicators.

interactions can disturb the satellite galaxy and strip gas from the satellite halo that could otherwise replenish star formation (e.g., Moore et al. 1996; Gunn \& Gott 1972; Kawata \& Mulchaey 2008). Many studies have found an increase in the fraction of red galaxies within halo virial radii (e.g., Dressler et al. 1997; Weinmann et al. 2006; Baxter et al. 2017). Wetzel et al. (2013) finds that the typical timescales for quenching are on the order of satellite orbital periods (2-4 Gyr), which matches the timescales for changes in the shape of the neighbour density distributions. Thus, we expect red and blue neighbours will correspond to long and short timescales since infall, respectively.

We perform our analyses on both red and blue neighbours around isolated hosts with $10.5<\log _{10}\left(M_{*} / M_{\odot}\right)<11.0$. We define these two bins using the $g-r$ colour distribution of all neighbours within our analysis area, i.e., $0.05-2.0 \mathrm{Mpc}$ from the isolated hosts (Fig. 5). We define blue neighbours as those with $0.0<g-r<0.75$ and red neighbours as those with $0.75<g-r<$ 1.0 .

\subsection{Simulation Data}

We use haloes from the Bolshoi-Planck dark matter simulation (Klypin et al. 2016a; Rodríguez-Puebla et al. 2016b), which followed a co-moving volume of $(250 \mathrm{Mpc} / h)^{3}$ with high mass resolution $\left(1.6 \times 10^{8} h^{-1} \mathrm{M}_{\odot}\right)$ and $2048^{3}$ particles $\left(\sim 8 \times 10^{9}\right)$. BolshoiPlanck adopted a flat $\Lambda$ CDM cosmology $\left(h=0.678, \Omega_{m}=0.307\right.$, $\sigma_{8}=0.823, n_{s}=0.96$ ); we also use this cosmology in our analysis. Halo finding and merger tree construction were done with Rockstar (Behroozi et al. 2013a) and Consistent Trees (Behroozi et al. 2013b), respectively. Following O'Donnell et al. (2021), halo accretion rates are derived from Bolshoi-Planck over the past dynamical time $t_{\mathrm{dyn}}=1 / \sqrt{G \rho_{\mathrm{vir}}}$. We use specific halo mass accretion rates,

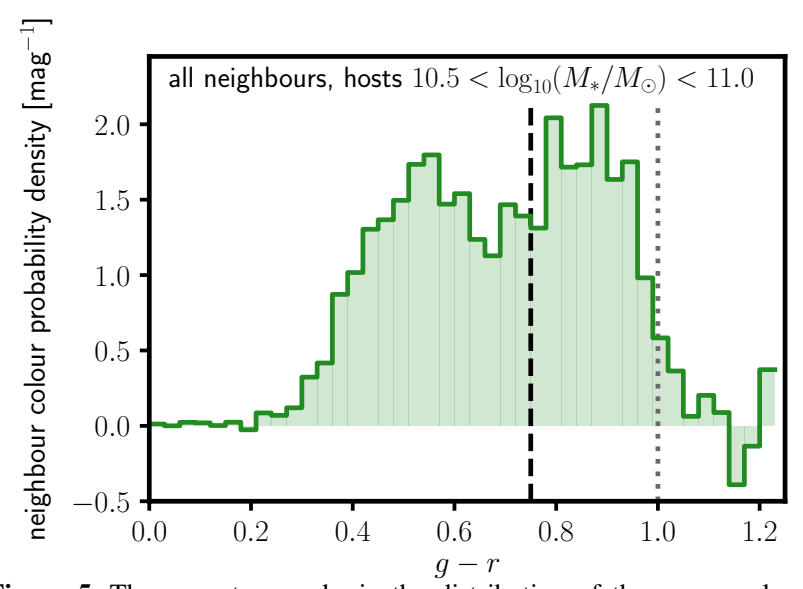

Figure 5. There are two peaks in the distribution of the $g-r$ colours of neighbouring galaxies between $0.05-2.0 \mathrm{Mpc}$ from isolated hosts with $10.5<\log _{10}\left(M_{*} / M_{\odot}\right)<11.0$. The dotted line indicates the $g-r<1.0$ colour cut applied to exclude photometric galaxies from higher redshifts. The dashed line at $g-r=0.75$ indicates the value used to separate red and blue neighbour galaxies.

which are normalised by halo virial masses, i.e.,

$\Gamma=\frac{\Delta \log \left(M_{\mathrm{vir}}\right)}{\Delta \log (a)} \equiv \frac{\log \left(\frac{M_{\mathrm{vir}}\left(t_{\text {now }}\right)}{M_{\mathrm{vir}}\left(t_{\text {now }}-t_{\mathrm{dyn}}\right)}\right)}{\log \left(\frac{a\left(t_{\text {now }}\right)}{a\left(t_{\text {now }}-t_{\text {dyn }}\right)}\right)}$,

following Diemer \& Kravtsov (2014). The distribution of these accretion rates only weakly depends on halo mass (Behroozi \& Silk 2015). In O'Donnell et al. (2021), we also used specific halo accretion rates calculated over the past $2 t_{\mathrm{dyn}}$ and found they were consistent with results using specific halo accretion rates over $1 t_{\text {dyn }}$ (Appendix B of O'Donnell et al. 2021).

For galaxy stellar masses, we use those from the UNIVERSEMA- 
CHINE empirical model (Behroozi et al. 2019), which implemented a Markov Chain Monte Carlo (MCMC) algorithm to model relationships between dark matter halo properties and galaxy properties (Behroozi et al. 2019). The UniverseMachine self-consistently constrained individual galaxies' properties to match observed stellar mass functions $(z \sim 0-4)$, specific star formation rates $(z \sim 0-8)$, cosmic star formation rates $(z \sim 0-10)$, UV luminosity functions $(z \sim 4-10)$, median UV-stellar mass relations $(z \sim 4-10)$, autoand cross-correlation functions $(z \sim 0-0.5)$, and quenched fractions $(z \sim 0-4)$. The UNIVERSEMACHINE constrained stellar masses at $z=0$ to match Moustakas et al. (2013) and used corrections from Bernardi et al. (2013) for extended galaxy profiles. Additionally, the UnIVERSEMACHINE allowed for orphans, i.e., it allowed satellites to persist after being destroyed in the dark matter simulation. Without including orphans, the model would predict a lower galaxy spatial correlation than is observed (see Appendix C of Behroozi et al. 2019 and §2.2.2. of Allen et al. 2019). In O’Donnell et al. (2021), we tested our results by excluding the orphan model and found that while it did slightly affect the neighbour density distributions close to the isolated hosts, it did not significantly alter our results (Appendix C of O'Donnell et al. 2021).

Following O'Donnell et al. (2021), we use galaxy positions and velocities from the UNIVERSEMACHINE. We also use observed stellar masses from the UnIVERSEMACHINE, which incorporate both random scatter and systematic offsets. While the UNIVERSEMACHINE also generates star formation rates, we discard this information to allow choosing SFRs that have different correlations with halo accretion rates.

\subsubsection{Sample Statistics}

As in O'Donnell et al. (2021), we combined catalogs from 14 simulation snapshots with $a=0.904$ to $a=1.002$. We identified isolated hosts following the same criteria as the observational data (no galaxy with a higher observed stellar mass within $2 \mathrm{Mpc}$ projected distance and $1000 \mathrm{~km} / \mathrm{s}$ velocity separation). Each snapshot had an average of 31026 isolated hosts with $10.5<\log _{10}\left(M_{*} / M_{\odot}\right)<11.0$ and 9541 isolated hosts with $11.0<\log _{10}\left(M_{*} / M_{\odot}\right)<11.5$. We note that $\gtrsim 94$ per cent of the isolated hosts were not satellites of larger haloes for both isolated host mass bins. ${ }^{2}$

To measure the uncertainties in the neighbour density distributions, we use jackknife sampling. We created 25 jackknife samples by averaging across the 14 snapshots with the same $50 \times 50 \mathrm{Mpc}$ region removed from each snapshot. Each jackknife sample has an average of $\sim 27000$ isolated hosts with $10.5<\log _{10}\left(M_{*} / M_{\odot}\right)<11.0$ and $\sim 9000$ isolated hosts with $11.0<\log _{10}\left(M_{*} / M_{\odot}\right)<11.5$. As noted in O'Donnell et al. (2021), the uncertainties for UNIVERSEMACHINE results differ from those for SDSS results because the background (noise) from the SDSS photometric data includes galaxies out to $z \sim 0.2$ (over $570 \mathrm{Mpc} / h$ ). However, the UniverseMachine simulation box is only $250 \mathrm{Mpc} / h$ per side.

\section{METHODS}

Our methodology follows the technique described in O'Donnell et al. (2021). Briefly, we identify isolated galaxies from SDSS spectroscopic data (Ahumada et al. 2019) with no larger neighbouring

2 The isolated galaxy catalogs are available at https://github.com/ caodonnell/DM_accretion galaxy within $2 \mathrm{Mpc}$ projected (on-sky) physical distance or 1000 $\mathrm{km} / \mathrm{s}$ velocity distance. We term these galaxies our isolated host sample. We calculate the density distribution of neighbouring galaxies using SDSS photometric data (Ahumada et al. 2019). To eliminate background and foreground contamination, for each isolated host, we create 100 random pointings that also follow our isolation criteria within the same sky footprint, and we subtract the neighbour density distribution around random pointings from the neighbour density distribution around our isolated hosts. We replicate this procedure in our simulation data from UNIVERSEMACHINE snapshots (Behroozi et al. 2019) by identifying isolated haloes, calculating the density of nearby neighbours, and subtracting background and foreground contamination by using 100 random pointings per isolated host.

Additionally, our methodology accounts for systematic biases in our data ( $\$ 2.3$ in O'Donnell et al. 2021). First, the stellar mass function from the UnIVERSEMACHINE differs from the stellar mass function in the SDSS MPA-JHU value-added catalogue due to different assumptions in converting luminosities to stellar masses; these result in the UNIVERSEMACHINE having more high-mass galaxies $\left(M_{*}>10^{11} M_{\odot}\right.$; Fig. 10 in O'Donnell et al. (2021)). We account for these differences by choosing analogous stellar mass cutoffs in the UniverseMachine such that the cumulative number density of galaxies with greater stellar masses matches that from the SDSS MPA-JHU catalogue (Table 1).

Second, the UniverseMachine assumes that the observed stellar masses of quiescent and star-forming galaxies have the same biases, but this may not be true in the real Universe given differences in metallicity, dust, and star formation histories between the two populations. These differences create a normalisation offset in the neighbour density distributions, though it should not affect the shapes of the distributions. We calculate this offset by matching the neighbour density distributions from SDSS and UNIVERSEMACHINE between 1.25-2.0 Mpc, as this region has the least correlation with accretion rates (O'Donnell et al. 2021). Table 2 lists typical values for these normalisation factors. In O'Donnell et al. (2021), we thoroughly tested the validity of this approach by comparing our observational results to simulation data from the UNIVERSEMACHINE where we selected simulated isolated hosts such that their density distribution normalisations matched the observed values ( $\$ 4.2$ of O'Donnell et al. 2021). We conducted this test using the same jackknife sampling across UNIVERSEMACHINE catalogs as is used in this paper, as well as using a single snapshot ( $a=0.956)$ with haloes from the Small MultiDark Planck simulation (SMDPL; Klypin et al. 2016b), which has a larger co-moving volume than the Bolshoi-Planck simulation $\left((400 \mathrm{Mpc} / h)^{3}\right.$ versus $(250 \mathrm{Mpc} / h)^{3}$, respectively). Both tests yielded consistent results with our approach applying calculated normalisation factors between the SDSS and UNIVERSEMACHINE neighbour density distributions. We also conducted additional tests assuming stellar mass offsets in the SDSS values (Appendix D1) and the UniverseMachine values (Appendix D2) to ensure the robustness of our analysis technique (O'Donnell et al. 2021).

Finally, to account for stellar mass completeness and background/foreground projection effects in SDSS, we weight the neighbour density distributions from SDSS data by

$w=z^{2} \times \frac{1}{V_{\max }\left(M_{*}\right)}$.

The factor of $z^{2}$ maximises signal-to-noise given Poisson variance in unassociated source counts (which scales as $z^{-2}$ ), and the factor $1 / V_{\max }\left(M_{*}\right)$ accounts for stellar mass completeness as computed 


\begin{tabular}{|c|c|c|c|}
\hline & $\begin{array}{c}\text { SDSS } \\
\log _{10}\left(M_{*} / M_{\odot}\right)\end{array}$ & $\begin{array}{l}\text { UNIVERSEMACHINE } \\
\log _{10}\left(M_{*} / M_{\odot}\right)\end{array}$ & $\begin{array}{c}\Phi\left(>M_{*}\right) \\
(\mathrm{Mpc} / h)^{-3}\end{array}$ \\
\hline \multirow{3}{*}{ 点 } & 10.50 & 10.50 & 0.64016 \\
\hline & 11.00 & 11.08 & 0.09464 \\
\hline & 11.50 & 11.75 & 0.00207 \\
\hline \multirow{4}{*}{$\begin{array}{l}\hat{\Xi} \\
\overline{0} \\
\frac{0}{50} \\
\overline{\vec{c}}\end{array}$} & 8.50 & 8.62 & 6.62222 \\
\hline & 9.00 & 8.93 & 4.85279 \\
\hline & 9.50 & 9.38 & 3.05361 \\
\hline & 10.00 & 9.93 & 1.62929 \\
\hline
\end{tabular}

Table 1. The SDSS MPA-JHU catalogue and UnIVERSEMAchine include different assumptions that affect their stellar mass functions. In our analysis, we use analogous stellar mass cutoffs in the UNIVERSEMACHINE such that the cumulative number density of more massive objects matches that from SDSS MPA-JHU. The first two columns summarises these stellar masses, and the third column indicates the cumulative number density of more massive galaxies. The first three rows are the limits for selecting isolated hosts, and the bottom four rows are the values for selecting nearby neighbours. Throughout this paper, we use stellar masses from the SDSS (first column).

\begin{tabular}{ccc}
$\begin{array}{c}\text { Isolated Host Mass } \\
{\left[\log _{10}\left(M_{*} / M_{\odot}\right)\right]}\end{array}$ & $\begin{array}{c}\text { Star-Formation } \\
\text { Indicator }\end{array}$ & $\begin{array}{c}\text { Normalisation Factor } \\
{[\mathrm{dex}]}\end{array}$ \\
\hline & $\mathrm{SSFR}>10^{-11} \mathrm{yr}^{-1}$ & $-0.226 \pm 0.206$ \\
$(10.5,11.0)$ & $\mathrm{SSFR}<10^{-11} \mathrm{yr}^{-1}$ & $-0.020 \pm 0.088$ \\
& $D_{n} 4000<1.6$ & $-0.199 \pm 0.208$ \\
& $D_{n} 4000>1.6$ & $-0.035 \pm 0.086$ \\
\hline & $\mathrm{SSFR}>10^{-11} \mathrm{yr}^{-1}$ & $-0.046 \pm 0.109$ \\
$(11.0,11.5)$ & $\mathrm{SSFR}<10^{-11} \mathrm{yr}^{-1}$ & $0.033 \pm 0.033$ \\
& $D_{n} 4000<1.6$ & $0.094 \pm 0.090$ \\
& $D_{n} 4000>1.6$ & $0.017 \pm 0.033$ \\
\hline
\end{tabular}

Table 2. Following O'Donnell et al. (2021), we apply a normalisation correction to match the neighbour density distributions between the UNIVERSEMACHINE and SDSS between 1.25-2.0 Mpc. This factor is required because the UNIVERSEMACHINE assumes the same biases between true and observed stellar masses for both star-forming and quiescent hosts. This table summarises the average normalisation factors between the observed SDSS neighbour density distributions and the UNIVERSEMACHINE predictions for no correlation $(\rho=0)$ between dark matter accretion rates and star formation rates. We include both star formation and quiescence indicators used in this paper. SSFR $>10^{-11} \mathrm{yr}^{-1}$ or $D_{n} 4000<1.6$ selects star-forming hosts, and SSFR $<10^{-11} \mathrm{yr}^{-1}$ or $D_{n} 4000>1.6$ selects quiescent hosts. For isolated hosts with $10.5<\log _{10}\left(M_{*} / M_{\odot}\right)<11.0$, we average the results for neighbour selection limits $\log _{10}\left(M_{*} / M_{\odot}\right)>10.0,9.5$, and 9.0 as we are only complete down to $\log _{10}\left(M_{*} / M_{\odot}\right)>8.95$ at the median host redshift $z=0.079$. For isolated hosts with $11.0<\log _{10}\left(M_{*} / M_{\odot}\right)<11.5$, we average the results for neighbour selection limits $\log _{10}\left(M_{*} / M_{\odot}\right)>10.0$ and 9.5 as we are only complete down to $\log _{10}\left(M_{*} / M_{\odot}\right)>9.30$ at the median host redshift $z=0.116$.

from Eq. 1. For a more detailed description of these weights, see $\S 2.3 .3$ of O'Donnell et al. (2021). We also reported in O'Donnell et al. (2021) that our results did not change if we weighted the distributions only by stellar mass completeness and exclude the inverse variance weights.

As we demonstrated in O'Donnell et al. (2021), the shapes of the neighbour density distributions encode information about $\rho$, the correlation between dark matter accretion and star formation. Specifically, the neighbour density distributions around highlyaccreting hosts steepen at a few hundred kpc, consistent with expectations that newly accreted dark matter is deposited at $\sim R_{200 \mathrm{~m}}$
(Wetzel \& Nagai 2015; Diemer et al. 2013). To quantify this shape and compare neighbour density distributions, we defined a shape parameter metric ( $\$ 2.2$ of O'Donnell et al. 2021):

$$
R=\frac{N \in\left(0.05 \mathrm{Mpc}-r_{\text {split }}\right)}{N \in\left(r_{\text {split }}-2.0 \mathrm{Mpc}\right)},
$$

which compares the number of neighbours close to isolated host galaxies versus the number of neighbours further away. The inner radius $(0.05 \mathrm{Mpc})$ conservatively excludes incompleteness from source blending in SDSS data, and the outer radius $(2.0 \mathrm{Mpc})$ is consistent with our isolation criterion. In O'Donnell et al. (2021), we also repeated our analysis with an even more conservative inner radius of $0.125 \mathrm{Mpc}$ and found that it did not significantly change our results.

We determined that $r_{\text {split }} \equiv 0.316 \mathrm{Mpc}$ maximises our sensitivity to differences between host halo dark matter accretion rates for star-forming and quiescent galaxies (O'Donnell et al. 2021). We quantify these differences using a shape ratio $R_{\mathrm{SF}} / R_{\mathrm{Q}}$ to compare the shape parameters of star-forming galaxies $\left(R_{\mathrm{SF}}\right)$ versus quiescent galaxies $\left(R_{\mathrm{Q}}\right)$. In our analysis of the SDSS data, we compute confidence levels by comparing the observed shape ratios to $R_{\mathrm{SF}} / R_{\mathrm{Q}} \equiv 1.0$, which is the expected shape ratio for no correlation $(\rho=0)$ between dark matter accretion and star formation activity.

To construct our dark matter accretion predictions, we bin isolated hosts from the UniverseMAchine simulation data (§ 2.2) by their specific halo accretion rates (Eq. 3) to match the fraction of star-forming versus quiescent hosts in SDSS. This procedure is described in detail in $\$ 3.3 .3$ of O'Donnell et al. (2021) and is summarised here in Fig. 6. Additionally, in O'Donnell et al. (2021), we showed that this procedure recovers the expected correlation strength $\rho$ between specific dark matter accretion rates and a host's status as star-forming or quiescent (Fig. 6 in O'Donnell et al. 2021). Briefly, we split the isolated host sample into 0.1 dex-wide bins of stellar mass to calculate the star-forming fraction, e.g., in the SDSS, for isolated hosts with $10.7<\log _{10}\left(M_{*} / M_{\odot}\right)<10.8,31$ per cent are star-forming based on having $D_{n} 4000<1$.6. We split the isolated hosts from the UnIVERSEMACHINE data into high- and low-accreting subsamples (based on their specific halo accretion rates) such that they match the star-forming fraction from SDSS for the relevant 0.1-dex bin of isolated host stellar mass. We then create 'star-forming' and 'quiescent' analogues using the correlation strength $\rho$ between halo accretion rates and star formation rates. For positive correlations, the star-forming analogues have a fraction $\rho$ of hosts randomly selected with replacement from the high-accreting subsample, and quiescent analogues have a fraction $\rho$ randomly selected with replacement from the low-accreting subsample. For negative correlations, the star-forming analogues have a fraction $|\rho|$ randomly selected with replacement from the low-accreting subsample, and the quiescent analogues have a fraction $|\rho|$ randomly selected with replacement from the high-accreting subsample. The remaining fraction of hosts in the analogues $(1-|\rho|)$ are randomly selected with replacement from all isolated hosts identified in the UNIVERSEMACHINE.

\section{RESULTS}

Below, we present results to test our choice of star formation indicator (\$4.1), results from splitting neighbouring galaxies into red and blue subsamples ( $\$ 4.2)$, and results from a higher isolated host mass range (\$4.3). In Appendix A, we include plots for other neighbour selection limits. 


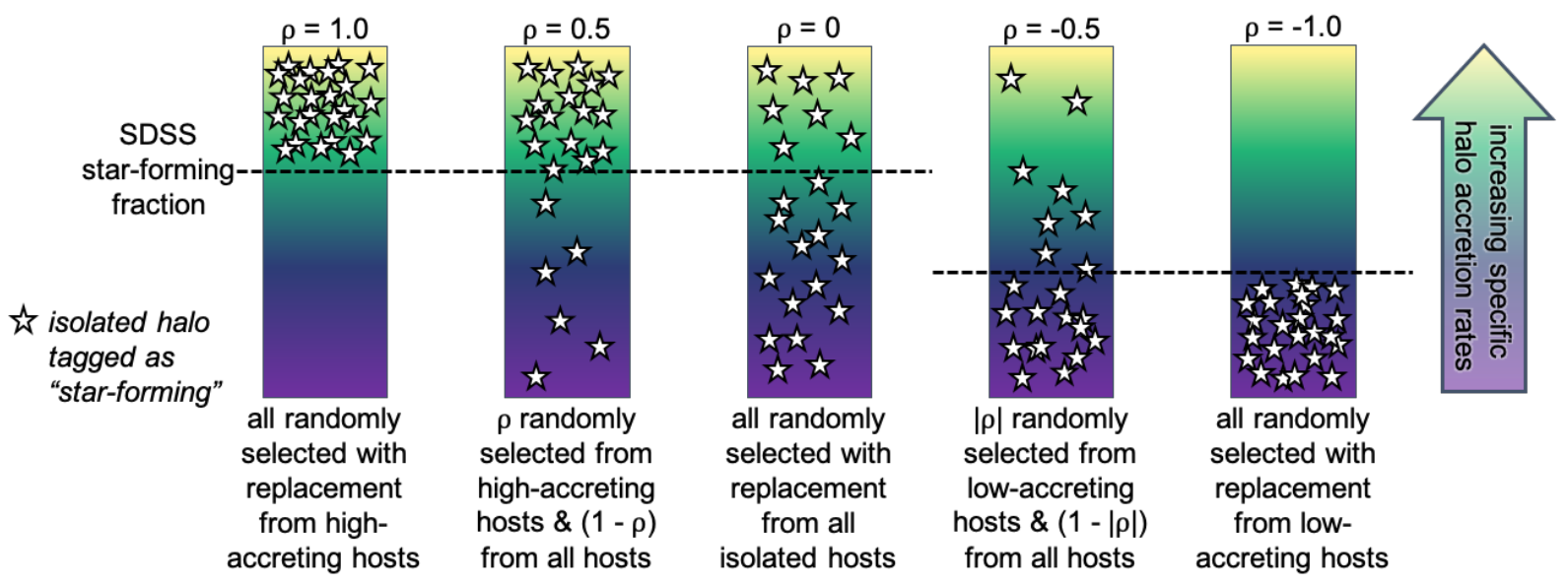

Figure 6. Schematic for generating analogues to the star-forming SDSS isolated hosts from UnIVERSEMACHINE data to test different correlation strengths $\rho$ between dark matter accretion and star formation. The coloured bars indicate all of the isolated hosts identified in the UNIVERSEMACHINE within a 0.1 dex stellar mass bin (e.g., $10.7<\log _{10}\left(M_{*} / M_{\odot}\right)<10.8$ ) sorted by increasing specific halo accretion rate (Eq. 3 ) from the bottom (purple) to the top (yellow) of the bars. The star icons in each example depict an isolated halo that is tagged as 'star-forming.' The dashed horizontal line indicates the corresponding star-formation fraction from the SDSS within the isolated host mass bin. For positive correlations $(\rho>0)$, this fraction is applied to identify the highest-accreting hosts; for negative correlations $(\rho<0)$, this fraction is applied to identify the lowest-accreting hosts. A similar strategy is used to create analogues to the quiescent isolated hosts from the SDSS.

\subsection{Star Formation \& Quiescence Indicators}

Fig. 7 compares the neighbour density distributions around isolated hosts with $10.5<\log _{10}\left(M_{*} / M_{\odot}\right)<11.0$ when binned by SSFRs versus $D_{n} 4000$ for the different neighbour mass selection limits. Since $\gtrsim 90$ per cent of isolated hosts were binned in the same way by the two indicators (Fig. 4), the resulting neighbour density distributions are also very similar. For both indicators, we see a dip in the neighbour density distribution for neighbours with higher masses $\left(M_{*} \gtrsim 10^{9.5} M_{\odot}\right)$ at $<0.1 \mathrm{Mpc}$ from the isolated hosts. We find consistent neighbour density distributions and shape ratios $\left(\Delta R_{\mathrm{SF}} / R_{\mathrm{Q}} \sim 0.2 \sigma\right)$ when binning the isolated hosts based on either SSFR or $D_{n} 4000$. Both indicators yield results that are consistent with non-positive correlations $(\rho \leq 0)$ between dark matter accretion and star formation at $\gtrsim 75$ per cent confidence.

\subsection{Neighbour Colours}

Table 3 reports the fraction of red neighbours (i.e., $0.75<g-r<$ 1.0) around isolated hosts with $10.5<\log _{10}\left(M_{*} / M_{\odot}\right)<11.0$. There is a small, though statistically insignificant, decrease in the fraction of red neighbours with $M_{*}>10^{10} M_{\odot}$ as the distance from the isolated host increases. The difference in the fraction of red neighbours around star-forming versus quiescent isolated hosts is not statistically different due to large uncertainties (\$2.1.1).

Fig. 8 shows that the neighbour density distributions of red and blue neighbours $(0.75<g-r<1.0$ and $0.0<g-r<0.75$, respectively) have similar shapes. For this analysis, we separate the isolated hosts into star-forming and quiescent bins based on their SSFRs; however, since SSFRs and $D_{n} 4000$ measurements track each other very closely (Fig. 4), the choice of star formation indicator does not yield significantly different results. Because of the minimum colour cutoff for red neighbours $(g-r>0.75)$, we limit our analysis to neighbours with $M_{*}>10^{9.5} M_{\odot}$ and $M_{*}>$ $10^{10} M_{\odot}$ to have sufficient signal-to-noise. The shape ratios (Fig. 9) are also similar $\left(\Delta R_{\mathrm{SF}} / R_{\mathrm{Q}} \sim 0.2 \sigma\right)$ and are consistent with nonpositive correlations $(\rho \leq 0)$ between dark matter accretion and star formation at $\gtrsim 90$ per cent confidence. Since we expect that red neighbours correspond to an older infall population (Wetzel et al. 2013, see also the discussion above in $\$ 2.1 .3$ ), these results would imply that the shape of the distribution is independent of the time since infall.

Furthermore, in O'Donnell et al. (2021), we noted that the neighbour density distributions with higher-mass neighbour selection limits had a deficit of neighbours close to the isolated hosts ( $\lesssim 1.25 \mathrm{kpc}$ ). In Fig. 8, the blue neighbour density distribution for the $M_{*}>10^{10} M_{\odot}$ selection shows this same deficit, as do the red neighbour density distributions around star-forming hosts for both the $M_{*}>10^{9.5} M_{\odot}$ and $M_{*}>10^{10} M_{\odot}$ selections. This deficit may be due to satellite galaxies being disrupted by their host galaxies more quickly than predicted in the UniverseMachine (e.g., Garrison-Kimmel et al. 2017).

\subsection{Host Stellar Masses}

Finally, we compare results with higher-mass isolated hosts $(11.0<$ $\left.\log _{10}\left(M_{*} / M_{\odot}\right)<11.5\right)$ using both star formation indicators. The rows of Fig.10 (as well as Fig. A1, A2, and A3 in Appendix A) compare across bins of isolated host mass. Fig. 11 summarises the results for both star formation indicators in the higher-mass isolated host bin. For both of our star formation indicators (SSFR and $\left.D_{n} 4000\right)$, results are similar $\left(R_{\mathrm{SF}} / R_{\mathrm{Q}}\right.$ are within $\left.\sim 0.2-0.3 \sigma\right)$ and remain consistent with correlations $\rho \leq 0.0$ between star formation and dark matter accretion at $\gtrsim 85$ per cent confidence. We also observe a deficit in higher-mass neighbours around isolated hosts from both stellar mass bins as compared to UNIVERSEMACHINE predictions, which may suggest that satellite galaxies are depleted by their host galaxies faster than expected (e.g., Garrison-Kimmel et al. 2017). 

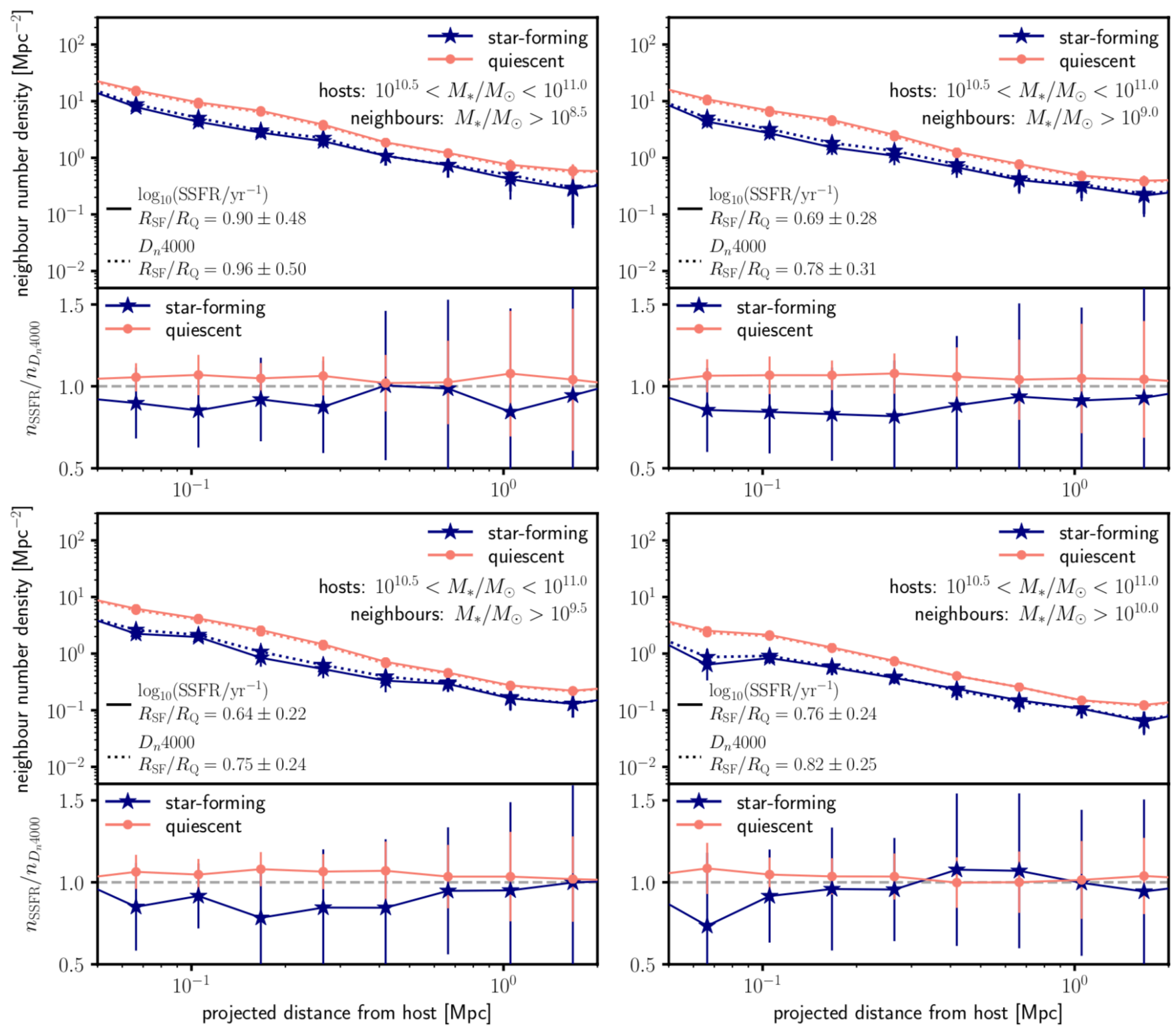

Figure 7. The neighbour density distributions around isolated hosts with $10.5<\log _{10}\left(M_{*} / M_{\odot}\right)<11.0$ are very similar when binning hosts by SSFRs or $D_{n} 4000$, due to the fact that the two indicators are highly correlated among our isolated host sample (Fig. 4). The four panels represent different neighbour mass selection limits. In each panel, the top plots compares the neighbour density distributions when binning isolated hosts by SSFR versus $D_{n} 4000$. The bottom plots shows the ratio of those distributions with a dashed horizontal line at $n_{\mathrm{SSFR}} / n_{D_{n} 4000}=1$ as a visual guide.

\section{DISCUSSION \& CONCLUSION}

We build on our work from O' Donnell et al. (2021), which presented a method to constrain the correlation strength between dark matter accretion and recent star formation (as determined by SSFRs) for Milky Way-mass galaxies at $z<0.123$ using the distribution of nearby neighbours. We found that our results favored non-positive correlations ( $~ 285$ per cent confidence). In this paper, we extend this analysis by

(i) comparing the density distributions of red versus blue neighbours, which trace older and more recent infall populations,

(ii) comparing the correlation between dark matter accretion and star formation when binning isolated hosts by $D_{n} 4000$ measure- ments, a longer-term quiescence indicator, versus binning isolated hosts by their specific star formation rates, and

(iii) analyzing higher-mass isolated hosts $(11.0<$ $\left.\log _{10}\left(M_{*} / M_{\odot}\right)<11.5\right)$ as an independent check of our results.

In all three cases, our results are consistent with non-positive correlations between dark matter accretion and star formation rates.

First, in O'Donnell et al. (2021), we noted that we would expect to find weak correlations if SSFRs change on timescales much shorter than satellite orbits ( $\left.\sim 2 t_{\text {dyn }} \sim 4 \mathrm{Gyr}\right)$. In this paper, we address this possible interpretation by (1) correlating dark matter accretion with $D_{n} 4000$, a long-term quiescence indicator; and (2) comparing red and blue populations of nearby neighbours, which trace satellite galaxy populations with different infall timescales. 

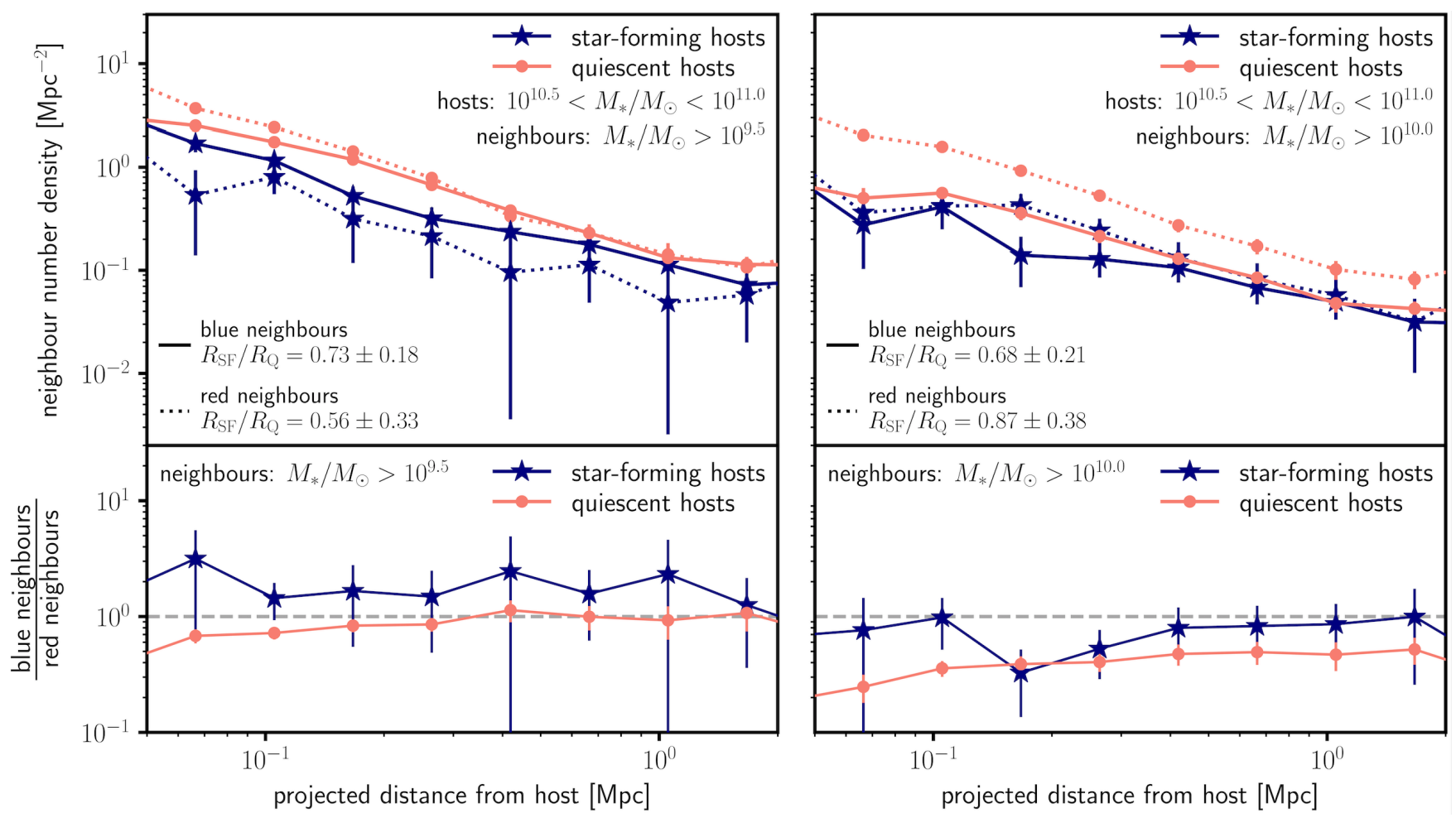

Figure 8. The neighbour density distributions around red and blue isolated hosts are very similar, suggesting that our finding of correlation strengths $\rho \leq 0$ (based on the measured shape ratios being $R_{\mathrm{SF}} / R_{\mathrm{Q}}<1$; see Fig. 9 below) between dark matter accretion and star formation applies to both recent and older infall populations as traced by blue and red neighbours, respectively. The star-forming and quiescent hosts are separated based on their SSFRs ( $\$ 2.1 .2)$. The top panels compare the neighbour density distributions of red and blue neighbours around isolated hosts with $10.5<\log _{10}\left(M_{*} / M_{\odot}\right)<11.0$, and the bottom row indicates the ratio of the blue neighbour density distribution to the red neighbour density distribution. In the bottom row, a horizontal dashed line at $n_{\text {blue }} / n_{\text {red }}=1.0$ is included as a visual guide for the slope of the ratio as a function of projected distance, although we do not necessarily expect the value of the observed ratio to equal 1 .

\begin{tabular}{cccc}
$\begin{array}{c}\text { Radial Range } \\
{[\mathrm{Mpc}]}\end{array}$ & \multicolumn{2}{c}{ Fraction of Red Neighbours } & Star-Forming Hosts \\
Quiescent Hosts & $\frac{f_{\text {red }}(\mathrm{Q})}{f_{\text {red }}(\mathrm{SF})}$ \\
\hline \hline \multicolumn{4}{c}{ Neighbours with $\boldsymbol{M}_{*}>10^{10} \boldsymbol{M}_{\odot}$} \\
\hline $0.05-0.316$ & $0.62 \pm 0.08$ & $0.70 \pm 0.02$ & $1.12 \pm 0.15$ \\
$0.316-1.00$ & $0.515 \pm 0.10$ & $0.66 \pm 0.03$ & $1.29 \pm 0.25$ \\
$0.316-2.00$ & $0.50 \pm 0.10$ & $0.67 \pm 0.03$ & $1.33 \pm 0.28$ \\
\hline \hline \multicolumn{4}{c}{ Neighbours with $M_{*}>10^{9.5} M_{\odot}$} \\
\hline $0.05-0.316$ & $0.32 \pm 0.11$ & $0.52 \pm 0.02$ & $1.63 \pm 0.58$ \\
$0.316-1.00$ & $0.26 \pm 0.16$ & $0.50 \pm 0.04$ & $1.93 \pm 1.23$ \\
$0.316-2.00$ & $0.32 \pm 0.17$ & $0.50 \pm 0.04$ & $1.57 \pm 0.85$ \\
\hline
\end{tabular}

Table 3. Our analysis does not have enough power to constrain differences in the fraction of red neighbours (i.e., $0.75<g-r<1.0$ ) around star-forming versus quiescent isolated hosts with stellar masses $10.5<\log _{10}\left(M_{*} / M_{\odot}\right)<11.0$. The star-forming and quiescent hosts are separated based on their SSFRs (\$2.1.2). The uncertainties are from our jackknife sampling of the SDSS data $(\$ 2.1 .1)$. We include data for neighbour mass selection limits of $\log _{10}\left(M_{*}\right)>9.5$ and 10.0; the lower-mass neighbour bins are noise-dominated due to few red neighbours passing these selection limits.

All of our results are consistent with our findings in O'Donnell et al. (2021) that generally rule out positive correlations between dark matter accretion and star formation within SDSS observational limits.

A second consideration is that neighbouring galaxies may be a biased tracer of the host galaxies' dark matter haloes. This concern remains in this paper's analysis; for example, this bias would affect all neighbours regardless of their $g-r$ colours. Additional measurements, such as weak lensing data, are needed to provide a different tracer of dark matter haloes to test the effect of systematic biases for using neighbouring galaxies to trace the density profile.

Our results are consistent with models that invoke modest recycling timescales for ejected gas, allowing for gas to quickly cool and re-accrete onto galaxies (e.g., Kereš et al. 2005; Dekel \& Birnboim 2006; Muratov et al. 2015; van de Voort 2017; Nelson et al. 2013, 2015). These models allow for new star formation at low redshifts even in the absence of new accretion. For haloes in our isolated host sample, only $\sim 20-30$ per cent of gas is converted into stars (Behroozi et al. 2019), which suggests there should be a large gas reservoir that could support further star formation. We also note the caveat that our findings would not necessarily require recycling processes if only a small fraction of accreted baryons need to be converted into star formation, thus decoupling the gas accretion from the fraction of gas that eventually gets converted into stars.

Additionally, our results remain consistent with observational studies that do not find strong positive correlations between halo growth and galaxy star formation in the recent Universe. For example, Tinker et al. (2017a) found only a small correlation between 


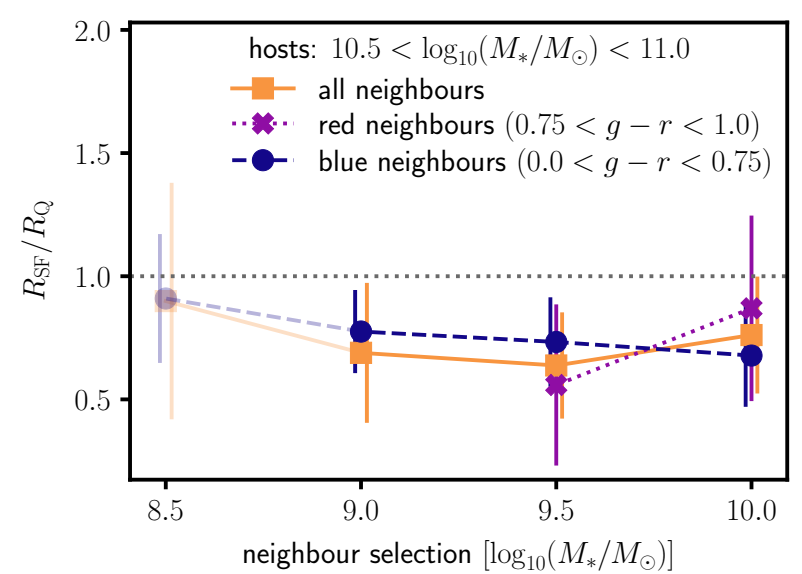

Figure 9. The shape ratio is not consistent with positive correlations between halo accretion rates and star formation regardless of nearby neighbour colours. Since we expect red neighbours may probe longer timescales than blue neighbours, these results likely mean that different infall populations are not affected in a significantly different manner by recent accretion for host galaxies in this mass range. The star-forming and quiescent hosts are separated based on their SSFRs (\$2.1.2). We only plot shape ratios for red neighbours $(0.75<g-r<1.0)$ for neighbours with $M_{*}>10^{9.5} M_{\odot}$ because the measurements for lower-mass neighbours are noise-dominated. The plot markers for the neighbour selection $M_{*}>10^{8.5} M_{\odot}$ are faded because neighbours of this stellar mass are not observable for all isolated hosts; for isolated hosts with $10.5<\log _{10}\left(M_{*} / M_{\odot}\right)<11.0$, at the median redshift $z=0.079$, the SDSS observation limit for neighbours is $M_{*}>10^{8.95} M_{\odot}$, and at the maximum redshift $z=0.123$, the SDSS observation limit for neighbours is $M_{*}>10^{9.36} M_{\odot}$.

the fraction of quenched central galaxies in galaxy groups and their local environmental density. As well, Behroozi et al. (2015) did not find a strong correlation between close galaxy pairs (a probe of major halo mergers) and star formation rates.

Our analysis of the neighbour populations did not have enough power to constrain the difference in the fraction of red versus blue neighbours around star-forming versus quiescent hosts (Table 3). Previous studies have found correlations between galaxy star formation rates, colours, and morphologies between satellites and host galaxies ('one-halo' conformity, e.g., Weinmann et al. 2006) as well as between galaxies separated at distances well beyond their virial radius ('two-halo' conformity, e.g., Kauffmann et al. 2013; Berti et al. 2017). However, Tinker et al. (2018) found that measurements of two-halo conformity may be due to satellite contamination. As our isolation criteria are extremely strict, the isolated host galaxies in our sample reside in different environments than most galaxies, and may show different conformity effects as a result (Hearin et al. 2016).

Future surveys with deeper photometric or spectroscopic limits may provide a better dataset for comparing density distributions of different neighbour populations to assess two-halo conformity among isolated central galaxies at $z=0$. If evidence for two-halo conformity existed at large distances (i.e., at distances well beyond $R_{\mathrm{vir}}$ ), our finding of non-positive correlations between dark matter halo accretion rates and star formation rates would have implications for the physical origin of galactic conformity. Hearin et al. (2016) found that galactic conformity could be driven by similar dark matter halo accretion rates between galaxies in the same largescale tidal environment, but this result relied on an assumption of a strong correlation between halo accretion rates and galactic star formation. If two-halo conformity is present among isolated central galaxies, that could suggest that a different process generates these correlations between galaxy colours, star formation rates, and other properties (e.g., Kauffmann 2015).

Finally, as noted in O'Donnell et al. (2021), future observational surveys, such as the Dark Energy Spectroscopic Instrument (DESI) Survey (DESI Collaboration et al. 2016), will allow for stronger constraints on the correlation between dark matter accretion and star formation. These surveys will detect a larger sample of isolated Milky Way-mass galaxies at higher redshifts, and thus generate a larger sample for this analysis. These data will also allow for measuring correlations between dark matter accretion and other host galaxy properties, such as metallicity, AGN activity, and velocity dispersion.

Furthermore, these surveys will have deeper photometric and spectroscopic limits, which will improve the analyses presented in this paper. For example, we will be able to perform this analysis on lower-mass isolated hosts. With the SDSS, we could only identify a small sample of isolated hosts with $10.0<\log _{10}\left(M_{*} / M_{\odot}\right)<10.5$, which were noise-dominated in their neighbour density distributions (§4.3). Additionally, we were limited to binning our isolated hosts into two bins ('star-forming' and 'quiescent'). It is possible that we may see different correlation strengths between dark matter accretion and star formation among galaxies with the strongest star formation than galaxies with weaker star formation. However, we are unable to complete this analysis with enough statistical significance to compare the populations. Surveys such as DESI will allow us to identify a larger sample of these hosts and therefore have a stronger signal to measure the shapes of their neighbour density distributions. In addition, deeper photometric limits will also allow us to detect more nearby neighbours. A larger sample of these galaxies will improve the signal-to-noise level when binning nearby neighbours by colour (§4.2) or other properties from SED fitting. These future results will provide stronger constraints on the relation between halo accretion and star formation within isolated host galaxies.

\section{ACKNOWLEDGEMENTS}

We thank Amanda Bauer, Gurtina Besla, Marla Geha, Elisabeth Krause, Dan Marrone, and Eduardo Rozo for helpful comments during the preparation of this paper.

Support for this research came partially via program number HST-AR-15631.001-A, provided through a grant from the Space Telescope Science Institute under NASA contract NAS5-26555. PB was partially funded by a Packard Fellowship, Grant \#2019-69646. An allocation of computer time from the UA Research Computing High Performance Computing (HPC) at the University of Arizona is gratefully acknowledged.

The coding and plots created for this work were done with Python packages NumPy (Oliphant 2015; van der Walt et al. 2011) and Matplotlib (Hunter 2007)

The Bolshoi simulations have been performed within the Bolshoi project of the University of California High-Performance AstroComputing Center (UC-HiPACC) and were run at the NASA Ames Research Center. Funding for the Sloan Digital Sky Survey IV has been provided by the Alfred P. Sloan Foundation, the U.S. Department of Energy Office of Science, and the Participating Institutions. SDSS-IV acknowledges support and resources from the Center for High-Performance Computing at the University of Utah. The SDSS web site is www.sdss.org. SDSS-IV is managed by the Astrophysical Research Consortium for the Participating Institutions of the SDSS Collaboration including the Brazilian Participa- 


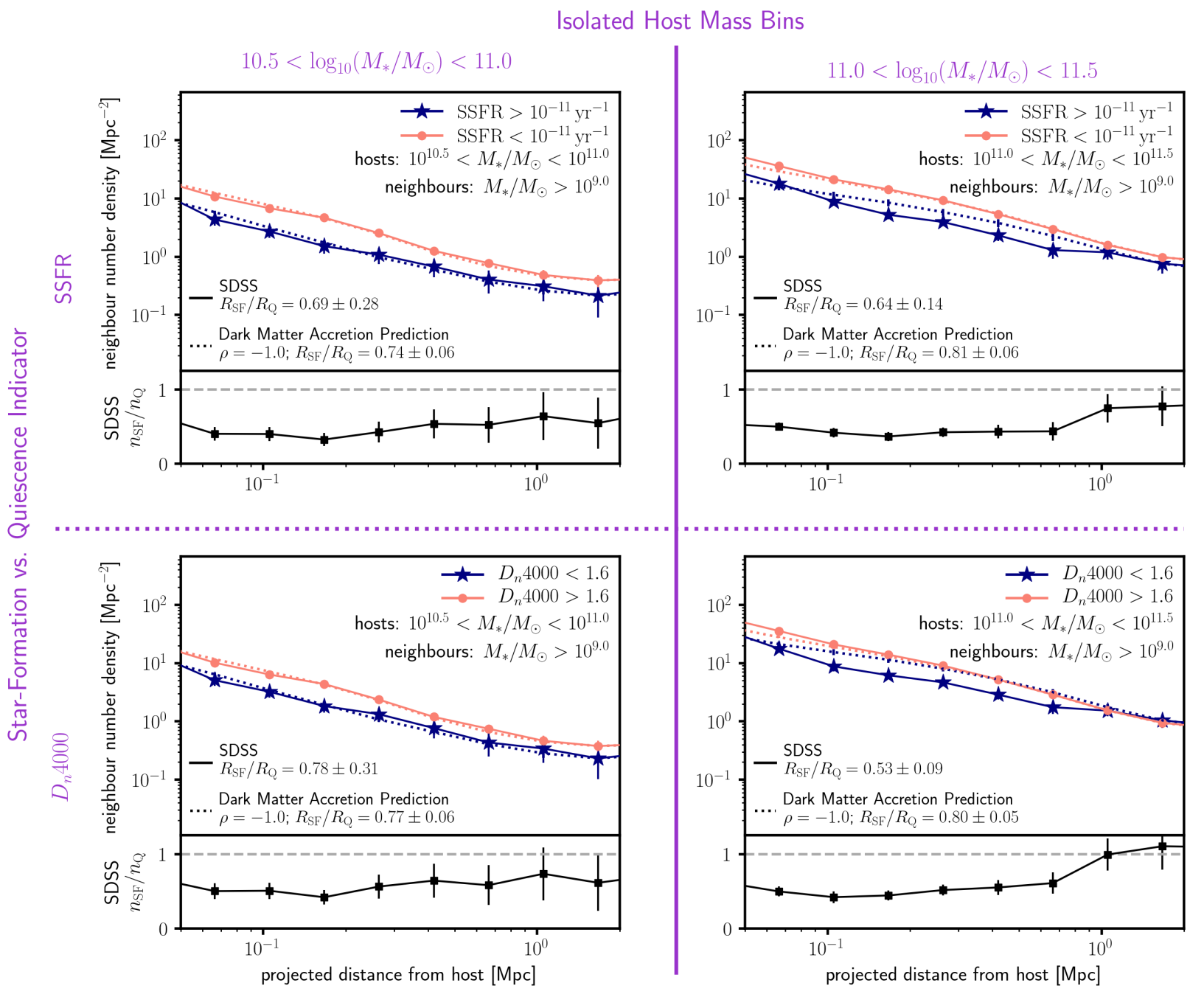

Figure 10. Our results are consistent with correlation strengths $\rho \leq 0$ between dark matter accretion and star formation regardless of isolated host mass bin (figure columns) or indicator to separate star-forming versus quiescent hosts (figure rows). This plot shows results with a neighbour mass selection $M_{*}>10^{9.0} M_{\odot}$; Appendix A includes plots for other neighbour $\boldsymbol{M}_{*}$ selection limits. In each panel, the top plots compare the neighbour density distributions from the SDSS to the UNIVERSEMACHINE predictions for anti-correlation $(\rho=-1)$ which is the closest match to the observed shape ratios. The bottom plots show the ratio of the observed neighbour density distributions for star-forming versus quiescent isolated hosts. A dashed horizontal line at $n_{\mathrm{SF}} / n_{\mathrm{Q}}=1$ is included as a visual guide to emphasise that the neighbour density distributions observed around star-forming hosts are flatter than the neighbour density distributions around quiescent hosts, which is consistent with non-positive correlations between dark matter accretion and star formation.

tion Group, the Carnegie Institution for Science, Carnegie Mellon University, the Chilean Participation Group, the French Participation Group, Harvard-Smithsonian Center for Astrophysics, Instituto de Astrofísica de Canarias, The Johns Hopkins University, Kavli Institute for the Physics and Mathematics of the Universe (IPMU) / University of Tokyo, the Korean Participation Group, Lawrence Berkeley National Laboratory, Leibniz Institut für Astrophysik Potsdam (AIP), Max-Planck-Institut für Astronomie (MPIA Heidelberg), Max-Planck-Institut für Astrophysik (MPA Garching), Max-Planck-Institut für Extraterrestrische Physik (MPE), National Astronomical Observatories of China, New Mexico State University, New York University, University of Notre Dame, Observatário Nacional / MCTI, The Ohio State University, Pennsylvania State University, Shanghai Astronomical Observatory, United Kingdom Participation Group, Universidad Nacional Autónoma de México, University of Arizona, University of Colorado Boulder, University of Oxford, University of Portsmouth, University of Utah, University of Virginia, University of Washington, University of Wisconsin, Vanderbilt University, and Yale University.

\section{DATA AVAILABILITY}

No new data were generated or analysed in support of this research. The isolated host catalogs derived from SDSS DR16 (Ahumada et al. 2019) and from the UniverseMachine (Behroozi et al. 2019) are available at https://github.com/caodonnell/DM_ accretion.

\section{REFERENCES}

Ahumada R., et al., 2019, arXiv e-prints, p. arXiv:1912.02905 


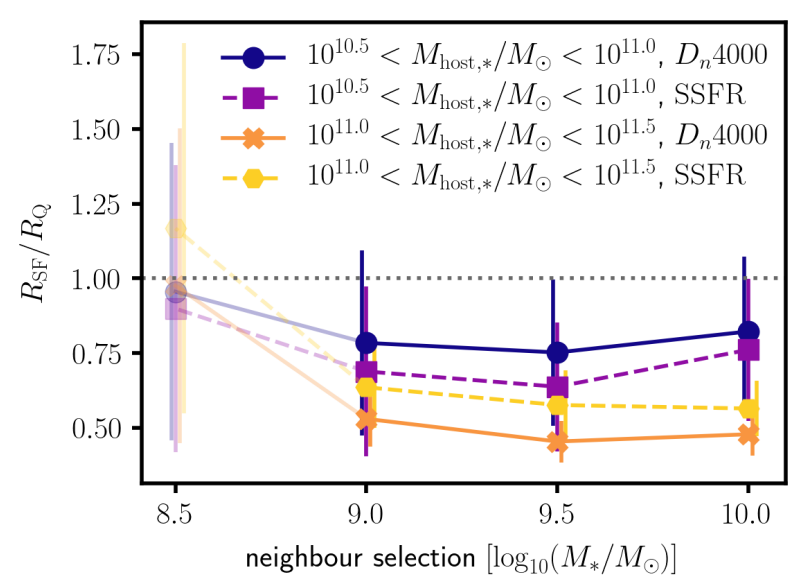

Figure 11. The shape ratios for our observed neighbour density distributions are all consistent with $\rho \leq 0$ regardless of choice of isolated host mass bin, indicator to separate star-forming versus quiescent hosts, and neighbour $M_{*}$ selection. As in Fig. 9, because we cannot observe neighbours with $M_{*} \sim 10^{8.5} M_{\odot}$ at all isolated host redshifts, those plot markers are shown with faded colours.

Allen M., Behroozi P., Ma C.-P., 2019, MNRAS, 488, 4916 Baldry I. K., et al., 2012, MNRAS, 421, 621

Balogh M. L., Morris S. L., Yee H. K. C., Carlberg R. G., Ellingson E., 1999, ApJ, 527, 54

Baxter E., et al., 2017, ApJ, 841, 18

Becker M. R., 2015, arXiv e-prints, p. arXiv:1507.03605

Behroozi P. S., Silk J., 2015, ApJ, 799, 32

Behroozi P. S., Wechsler R. H., Wu H.-Y., 2013a, ApJ, 762, 109

Behroozi P. S., Wechsler R. H., Wu H.-Y., Busha M. T., Klypin A. A., Primack J. R., 2013b, ApJ, 763, 18

Behroozi P. S., et al., 2015, MNRAS, 450, 1546

Behroozi P., Wechsler R. H., Hearin A. P., Conroy C., 2019, MNRAS, p. 1134

Bell E. F., McIntosh D. H., Katz N., Weinberg M. D., 2003, The Astrophysical Journal Supplement Series, 149, 289

Bernardi M., Meert A., Sheth R. K., Vikram V., Huertas-Company M., Mei S., Shankar F., 2013, MNRAS, 436, 697

Berti A. M., Coil A. L., Behroozi P. S., Eisenstein D. J., Bray A. D., Cool R. J., Moustakas J., 2017, ApJ, 834, 87

Berti A. M., Coil A. L., Hearin A. P., Behroozi P. S., 2021, AJ, 161, 49

Blanton M. R., et al., 2005, AJ, 129, 2562

Blanton M. R., Kazin E., Muna D., Weaver B. A., Price-Whelan A., 2011, AJ, 142, 31

Brinchmann J., Charlot S., White S. D. M., Tremonti C., Kauffmann G., Heckman T., Brinkmann J., 2004, MNRAS, 351, 1151

Chabrier G., 2003, PASP, 115, 763

Cohn J. D., 2017, MNRAS, 466, 2718

DESI Collaboration et al., 2016, arXiv e-prints, p. arXiv:1611.00036

Deason A. J., Fattahi A., Frenk C. S., Grand R. J. J., Oman K. A., GarrisonKimmel S., Simpson C. M., Navarro J. F., 2020, arXiv e-prints, p. arXiv:2002.09497

Dekel A., Birnboim Y., 2006, MNRAS, 368, 2

Diemer B., Kravtsov A. V., 2014, ApJ, 789, 1

Diemer B., More S., Kravtsov A. V., 2013, ApJ, 766, 25

Dressler A., et al., 1997, ApJ, 490, 577

Garrison-Kimmel S., et al., 2017, MNRAS, 471, 1709

Geha M., Blanton M. R., Yan R., Tinker J. L., 2012, ApJ, 757, 85

Gunn J. E., Gott J. Richard I., 1972, ApJ, 176, 1

Hearin A. P., Behroozi P. S., van den Bosch F. C., 2016, MNRAS, 461, 2135

Hunter J. D., 2007, Computing in Science Engineering, 9, 90

Kauffmann G., 2015, MNRAS, 454, 1840

Kauffmann G., et al., 2003, MNRAS, 341, 33

Kauffmann G., Li C., Zhang W., Weinmann S., 2013, MNRAS, 430, 1447
Kawata D., Mulchaey J. S., 2008, ApJ, 672, L103

Kereš D., Katz N., Weinberg D. H., Davé R., 2005, MNRAS, 363, 2

Klypin A., Yepes G., Gottlöber S., Prada F., Heß S., 2016a, MNRAS, 457, 4340

Klypin A., Yepes G., Gottlöber S., Prada F., Heß S., 2016b, MNRAS, 457, 4340

Lee C. T., Primack J. R., Behroozi P., Rodríguez-Puebla A., Hellinger D., Dekel A., 2017, MNRAS, 466, 3834

Moore B., Katz N., Lake G., Dressler A., Oemler A., 1996, Nature, 379, 613

More S., Diemer B., Kravtsov A. V., 2015, ApJ, 810, 36

More S., et al., 2016, ApJ, 825, 39

Moster B. P., Naab T., White S. D. M., 2018, MNRAS, 477, 1822

Moustakas J., et al., 2013, ApJ, 767, 50

Muratov A. L., Kereš D., Faucher-Giguère C.-A., Hopkins P. F., Quataert E., Murray N., 2015, MNRAS, 454, 2691

Nelson D., Vogelsberger M., Genel S., Sijacki D., Kereš D., Springel V., Hernquist L., 2013, MNRAS, 429, 3353

Nelson D., Genel S., Vogelsberger M., Springel V., Sijacki D., Torrey P., Hernquist L., 2015, MNRAS, 448, 59

O'Donnell C., Behroozi P., More S., 2021, MNRAS, 501, 1253

Oliphant T. E., 2015, Guide to NumPy, 2nd edn. CreateSpace Independent Publishing Platform, North Charleston, SC, USA

Planck Collaboration et al., 2018, arXiv e-prints, p. arXiv:1807.06209

Rodríguez-Puebla A., Primack J. R., Behroozi P., Faber S. M., 2016a, MNRAS, 455, 2592

Rodríguez-Puebla A., Behroozi P., Primack J., Klypin A., Lee C., Hellinger D., 2016b, MNRAS, 462, 893

Rykoff E. S., et al., 2014, ApJ, 785, 104

Somerville R. S., Davé R., 2015, ARA\&A, 53, 51

Tinker J. L., Wetzel A. R., Conroy C., Mao Y.-Y., 2017a, MNRAS, 472, 2504

Tinker J. L., et al., 2017b, ApJ, 839, 121

Tinker J. L., Hahn C., Mao Y.-Y., Wetzel A. R., Conroy C., 2018, MNRAS, 477, 935

Wechsler R. H., Tinker J. L., 2018, ARA\&A, 56, 435

Weinmann S. M., van den Bosch F. C., Yang X., Mo H. J., 2006, MNRAS, 366,2

Wetzel A. R., Nagai D., 2015, ApJ, 808, 40

Wetzel A. R., Tinker J. L., Conroy C., 2012, MNRAS, 424, 232

Wetzel A. R., Tinker J. L., Conroy C., van den Bosch F. C., 2013, MNRAS, 432, 336

van de Voort F., 2017, in Fox A., Davé R., eds, Astrophysics and Space Science Library, Vol. 430, Gas Accretion onto Galaxies. Springer, p. 301, doi:10.1007/978-3-319-52512-9_13

van der Walt S., Colbert S. C., Varoquaux G., 2011, Computing in Science Engineering, 13, 22

This paper has been typeset from a $\mathrm{T}_{\mathrm{E}} \mathrm{X} / \mathrm{L} \mathrm{T} \mathrm{E} \mathrm{X}$ file prepared by the author.

\section{APPENDIX A: DENSITY DISTRIBUTIONS FOR ADDITIONAL NEIGHBOUR SELECTION LIMITS}

Below, we present the neighbour density distributions around isolated hosts from both mass bins $\left(10.5<\log _{10}\left(M_{*} / M_{\odot}\right)<11.0\right.$ and $11.0<$ $\left.\log _{10}\left(M_{*} / M_{\odot}\right)<11.5\right)$ and both indicators used to separate star-forming and quiescent hosts (SSFR and $D_{n} 4000$ ) for the neighbour mass selections not included in Fig.10 $\left(M_{*}>10^{8.5} M_{\odot},>10^{9.5} M_{\odot}\right.$, and $\left.>10^{10.0} M_{\odot}\right)$. These plots follow the same plot styles as Fig. 10, and all are consistent with correlation strengths $\rho \leq 0$ between dark matter accretion and star formation (Fig. 11). 


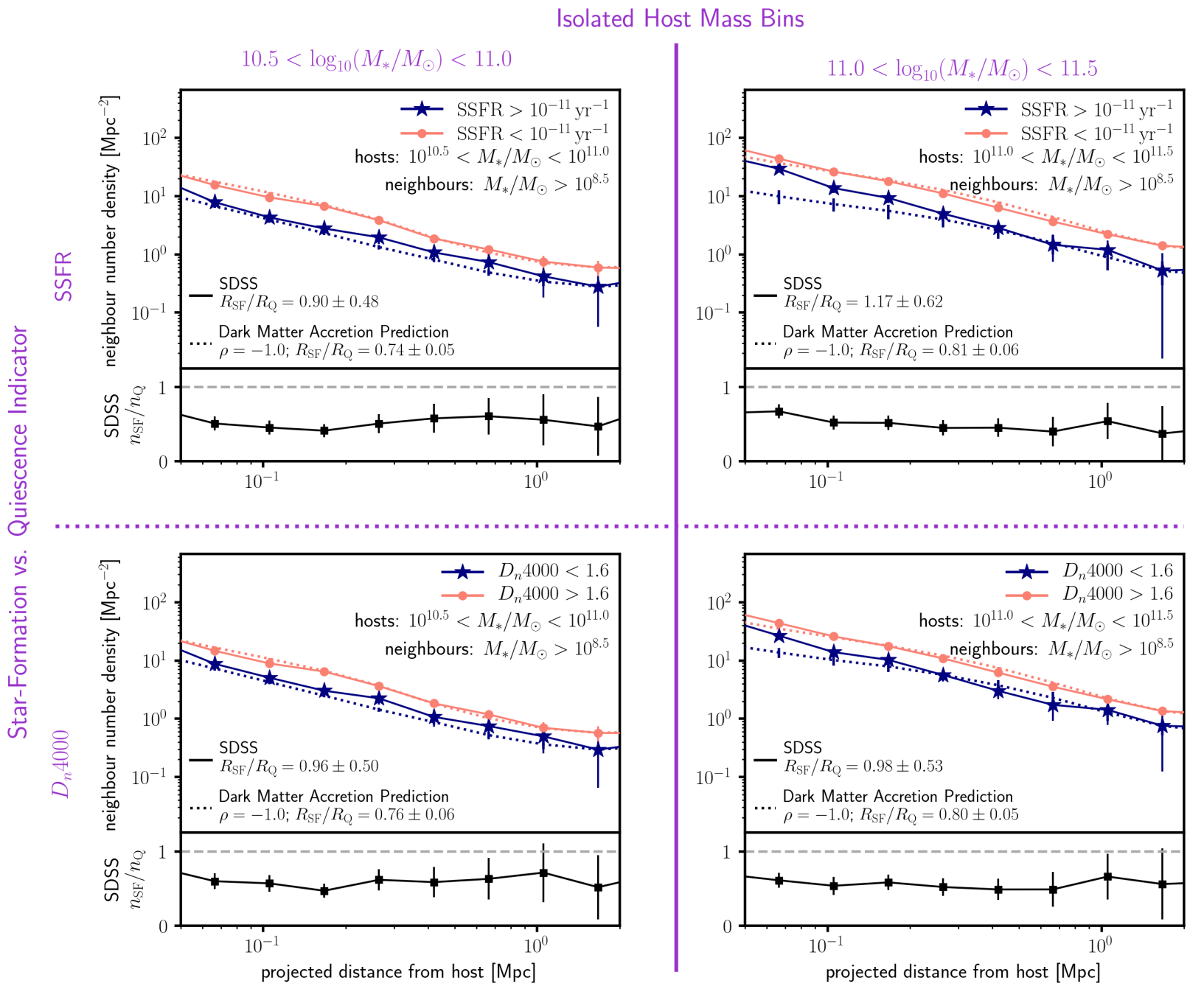

Figure A1. Same as Fig. 10, but with neighbours with $M_{*}>10^{8.5} M_{\odot}$. We note that neighbours at these lower masses are not observable in SDSS throughout the isolated host redshift range. For isolated hosts with $10.5<\log _{10}\left(M_{*} / M_{\odot}\right)<11.0$, the SDSS observation limits for are $M_{*}>10^{8.95} M_{\odot}$ at the median redshift $z=0.079$ and $M_{*}>10^{9.36} M_{\odot}$ at the maximum redshift $z=0.123$. Similarly, for isolated hosts with $11.0<\log _{10}\left(M_{*} / M_{\odot}\right)<11.5$, the SDSS observation limits are $M_{*}>10^{9.30} M_{\odot}$ at the median redshift $z=0.116$ and $M_{*}>10^{10.4} M_{\odot}$ at the maximum redshift $z=0.183$. 


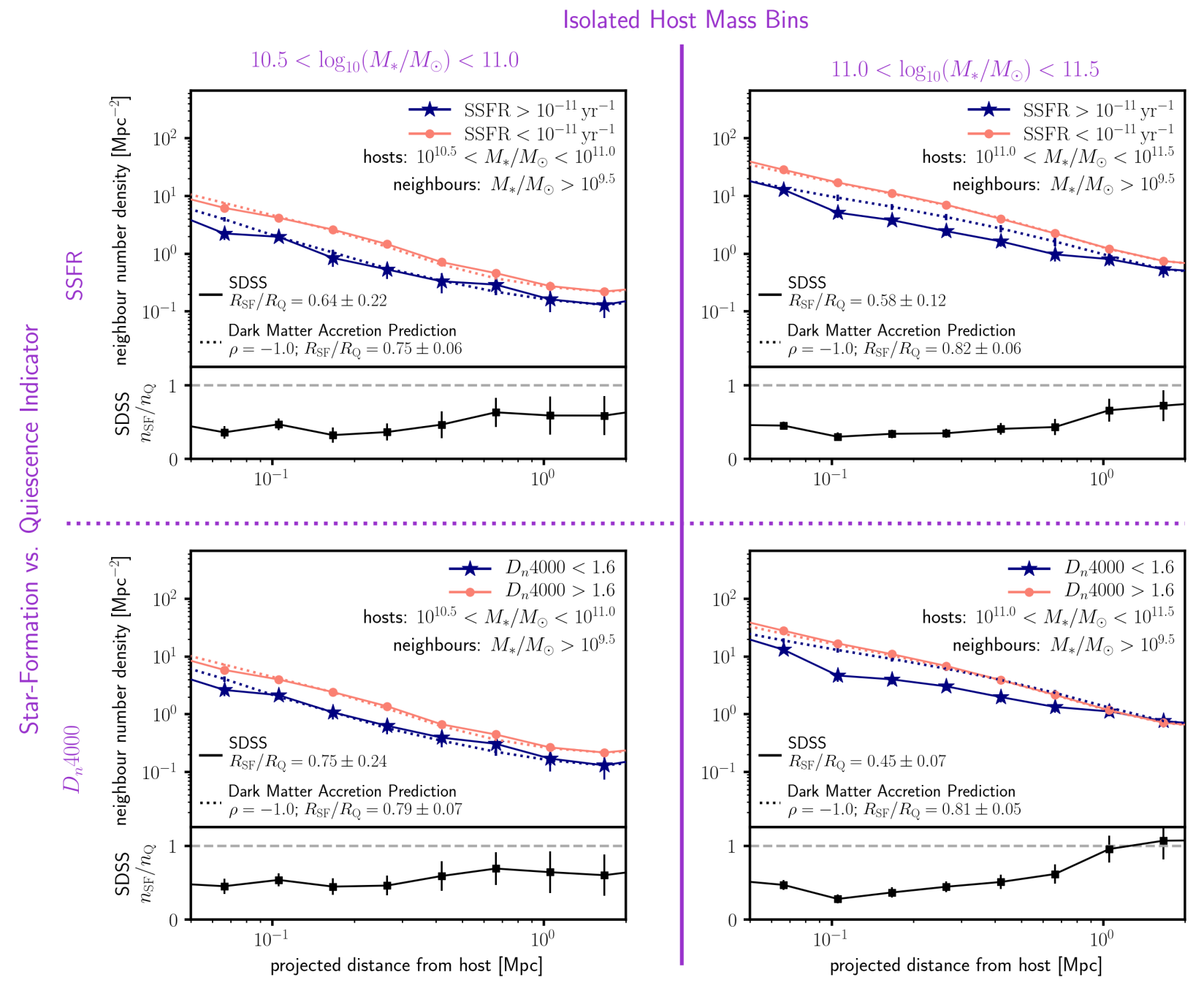

Figure A2. Same as Fig. 10, but with neighbours with $M_{*}>10^{9.5} \boldsymbol{M}_{\odot}$. These neighbour density distributions are also consistent with $\rho \leq 0.0$ with $\gtrsim 85$ per cent confidence. 


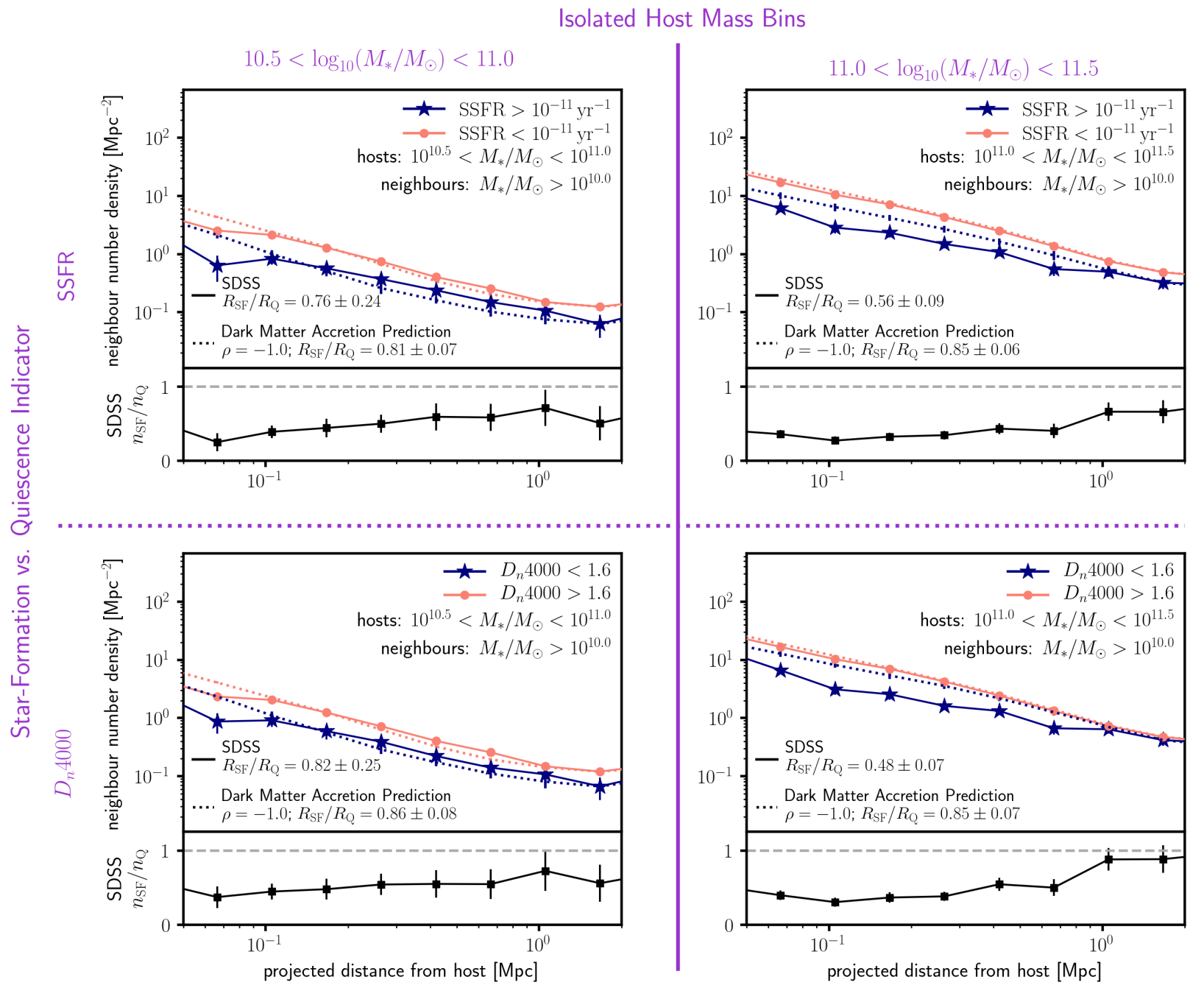

Figure A3. Same as Fig. 10, but with neighbours with $M_{*}>10^{10.0} \boldsymbol{M}_{\odot}$. These neighbour density distributions are also consistent with $\rho \leq 0.0$ with $\gtrsim 85$ per cent confidence. 